\title{
Flight Demonstration of Autonomous Noncooperative Rendezvous in Low Earth Orbit
}

\author{
Gabriella Gaias Jean-Sébastien Ardaens
}

January 17, 2018

This is a postprint version of the paper published by the American Institute of Aeronautics and Astronautics in Journal of Guidance, Control, and Dynamics (first online November 16, 2017).

Link: https://arc.aiaa.org/doi/abs/10.2514/1.G003239

DOI: 10.2514/1.G003239.

Cite this article as: Gaias, Gabriella and Ardaens, Jean-Sbastien (2017) Flight Demonstration of Autonomous Noncooperative Rendezvous in Low Earth Orbit. Journal of Guidance, Control, and Dynamics.

\begin{abstract}
This paper presents ultimate design, implementation, and in-flight performance of the spaceborne guidance navigation and control system which enabled the Autonomous Vision Approach Navigation and Target Identification (AVANTI) experiment; a flight demonstration developed by the German Space Operations Center (GSOC) of the German Aerospace Center (DLR) and carried out in November 2016. Designed to prove the viability to perform far- to mid-range proximity operations with respect to a noncooperative flying object using only optical angle measurements, AVANTI realized the first autonomous vision-based rendezvous to a passive target spacecraft in low Earth orbit. Within this experiment, the DLR Earth-observation BIROS satellite approached down to less than $50 \mathrm{~m}$ of inter-satellite distance the BEESAT-4 CubeSat, previously released in orbit by BIROS itself. To this end, a dedicated spaceborne formation-flying system carried out relative navigation and maneuver planning tasks. Moreover, it took over BIROS orientation and maneuvering capabilities to steer the spacecraft along a passively safe rendezvous trajectory. During AVANTI, the images taken by BIROS constituted the only source of relative navigation information. In the absence of external, independent, and precise navigation data of the target satellite, AVANTI performances have been assessed against the ground-based post-facto reprocessing of the images collected in flight.
\end{abstract}




\section{Introduction}

The broadly shared interest of increasing the technology readiness level of noncooperative rendezvous in low Earth orbits (LEO) is motivated by the need to enable unmanned on-orbit servicing (OOS) missions, devoted to realize strategic applications like debris-removal tasks. The absence of cooperation between chaser satellite and target object (e.g., no inter-satellite communication, no active markers on the target), affects the choice of sensors able to provide observations for relative navigation purposes. In this frame, a fully vision-based approach constitutes an appealing low-cost option since it exploits passive sensors (e.g., optical or infrared cameras), employable at various inter-satellite separation ranges, with little mass/power impact on the design of the chaser spacecraft. The limit-case of such minimalistic design strategy is to rely exclusively on an optical monocular camera to reach the close-range region to the target body, where of course a more comprehensive assembly of sensors is required to prepare and carry out contact interaction phases. Hence, the Autonomous Vision Approach Navigation and Target Identification (AVANTI) experiment has been the in-flight demonstration of the feasibility of such aforementioned limit-case, since the star-tracker, already onboard on BIROS to fulfill the conventional attitude determination task, has been re-used as far-range camera to provide relative navigation observations [1, 2]. As long as it is acceptable to approximate the center of mass of the target object with the intensity centroid of the luminous spot, optical measurements are the two angles subtended to the line-of-sight (LOS) to the imaged target. Nevertheless, this simple approach, requiring an elementary spacecraft design, comes at the cost of a weakly observable relative navigation problem (i.e., lack of direct measure of the relative range). Or equivalently, complexity shifts from the spacecraft design to the development of guidance navigation and control (GNC) algorithms able to achieve a practicable solution, given sensors and actuators in-flight performance.

Some remarkable experiences of spacecraft rendezvous based on anglesonly measurements have been performed so far in LEO by different industrial companies and research institutions. It is believed that the first activities took place within Orbital Express, a U.S.'s Defense Advanced Research Projects Agency mission, in 2007. Here a prototype servicing satellite accomplished autonomous rendezvous, capture, maintenance, and servicing of a surrogate next-generation serviceable satellite employing its on-purposedeveloped sensors' assembly [3]. In addition to these primary OOS goals, a noncooperative autonomous approach starting at $7 \mathrm{~km}$ distance has been carried out guiding the servicing spacecraft mainly with passive optical and infrared imaging [4]. Nevertheless, due to the lack of published technical details, it is impossible to assess to what extent optical LOS measurements have been complemented by the other available sources of navigation data. 
The remaining flight heritage has been collected on the Prototype Research Instruments and Space Mission Technology Advancement (PRISMA) formation flying test bed, taking advantage from the vision based sensor (VBS) of the Danish Technical University (DTU) [5] embarked on the active Mango spacecraft. This VBS system presented two supplementary camera heads (i.e., one far-range, one close-range) in addition to the pair of star-tracker heads in charge of supporting the attitude determination. In April 2011, OHB-Sweden, prime contractor of PRISMA, executed a LOS-based noncooperative rendezvous from $30 \mathrm{~km}$ to $50 \mathrm{~m}$ of separation in the frame of the Autonomous Rendezvous (ARV) experiment [6]. In this occasion, after a first phase of target searching lost-in-space function at $30 \mathrm{~km}$ distance, a leader-follower formation has been established without reducing the along-track separation, thus fostering the convergence of the relative navigation filter with the help of large out-of-plane maneuvers. This stage was then followed by a hopping V-bar approach, by means of the onboard guidance and control system. Successively, in August 2011, at conclusion of the PRISMA operations session in the DLR/GSOC premises [7], GSOC operated the Mango spacecraft to re-establish a close formation (i.e., at circa $4 \mathrm{~km}$ of inter-satellite distance) after that the spacecraft got separated by more than $60 \mathrm{~km}$. Within this activity, named Formation Re-Acquisition phase [8], two-line-elements (TLE) have been simply refined in the crosstrack plane with the solution coming from a prototype LOS-based navigation filter that processed on-ground 5-hours slots of pictures per day. Afterwards, during the extended phase of the PRISMA mission, both the French Aerospace Agency (CNES) and DLR/GSOC performed further activities. CNES carried out a dedicated campaign in October/November 2011 including four rendezvous (either starting from 4 or $10 \mathrm{~km}$ of separation) having $3 \mathrm{~m} / \mathrm{s}$ of delta-v available 9 . Here, the newly updated onboard navigation system exploited the VBS LOS output to feed the onboard controller, in charge to track some (in-plane) way-points provided from ground. Finally, in April 2012, DLR/GSOC performed the Advanced Rendezvous Demonstration using Global Positioning System and Optical Navigation (ARGON) experiment [10]. In this occasion, a dedicated ground-based flight dynamics system has been developed for routine processing of the camera images collected onboard, for estimating the relative orbit, and for maneuvering towards the target. As a result, an overall approach from 30 to $3 \mathrm{~km}$ has been accomplished over five days.

Besides these in-flight activities, it is worthy to mention the Iterative Reduction of Inspection Distance with Embedded Safety (IRIDES) experiment proposed by OHB-Sweden for the conclusive phase of PRISMA [11. Its objective was to use the remaining delta-v available to approach a truly noncooperative target (i.e., the French Picard satellite) and to collect closerange images for future OOS research. Major challenge was the mission analysis study and transfer phase to reach Picard's orbit [12]. As for the 
rendezvous part, a far-range approach was foreseen, followed by inspection phases through passively safe spiraling relative orbits. Hence, a similar principle adopted within AVANTI, though, in this case, facilitated by the absence of eclipses (i.e., dusk-dawn orbit) and by the negligible action of differential aerodynamic drag. Nevertheless, the rendezvous stage of the IRIDES experiment could never begin due to the delta-v depletion occurred in July 2014 [13.

As for the LOS-based-only flight demonstrations accomplished so far, AVANTI expands on the current state-of-the-art regarding the following fundamental aspects:

1. the AVANTI demonstration has been performed within a truly noncooperative scenario, since during the experiment execution no target tracking data were available, except for the images taken by the farrange camera. Contrarily, PRISMA was a cooperative test bed which, aside from its primary cooperative formation-flying objectives (e.g., GPS-based, radio frequency RF-based), has been used to mimic a noncooperative scenario. This structural difference impacts: safety management and design of the guidance policy. Regarding the first point, every activity on PRISMA benefited from the continual support of a GPS-based collision avoidance layer in real-time, onboard [14] and on-ground during contacts. Secondly, and consequently, in-plane only approaching trajectories, as performed by ARV and CNES, would be extremely risky if relying exclusively on optical LOS measurements and/or in the presence of failures leading to the interruption of the maneuvering activity (e.g., s/c contingencies, thruster system failures). Albeit ARGON already introduced the concept of spiraling trajectory, the conservation of a certain relative distance normal to the flight direction (i.e., prerequisite to guarantee collision avoidance via passive safety) has been enforced only for the final, bounded, target orbit. AVANTI firstly applied the one-orbit-minimum cross-track distance criterion throughout all rendezvous phases. And, to this end, it extended such principle to drifting relative orbits, resulting from nonvanishing relative semi-major axis encountered during a rendezvous or produced by the action of the differential aerodynamic drag [15].

2. the onboard GNC algorithms employed for AVANTI are developed from an advanced model of relative dynamics for near-circular lowEarth orbits: parametrized in relative orbital elements (ROEs), accurate also at large distances (i.e., more than $30 \mathrm{~km}$ ), and including mean effects of $J_{2}$ and time-varying non-conservative perturbations like differential aerodynamic drag [16]. Both OHB-Sweden and CNES flight algorithms, instead, exploited Cartesian coordinates (i.e., inaccurate for separations larger than few kilometers) and the YamanakaAnkersen state transition matrix [17] (i.e., not including perturba- 
tions), even though PRISMA presented a perturbed almost-circular orbit scenario. The flight dynamics system of ARGON, on the other hand, made use of the ROE-based formulation developed to support spaceborne precise formation-keeping applications (i.e., Spaceborne Autonomous Formation Flying Experiment [18] and TanDEM-X Autonomous Formation Flying system [19]). This included only mean $J_{2}$ effects on almost-bounded relative orbits; thus suboptimal for large reconfigurations and/or in environments with considerable variations of the relative semi-major axis. In addition to completeness, accuracy, and geometrical significance of the ROE formulation, the relative dynamics modeling exploited in AVANTI presents a practical compact form, that lead to the development of several semi-analytical expressions, which served as backbone to the flight algorithms.

3. AVANTI has implemented an innovative, fully autonomous, guidance strategy to tackle large relative orbit reconfigurations over extended time frames, such as the far- to close-range rendezvous problem. Such strategy aims at minimizing the delta-v cost to reach a target relative state at a given time, while supporting the definition of user-defined time constraints, and producing a solution continuously compliant with the aforementioned passive-safety criterion [20]. Thus, from a practical point of view, the chaser spacecraft can cope with phases where maneuvers are inhibited. In addition, each transfer profile can be at any time interrupted without leading to any collision between chaser and target object. This methodology is based on planning the relative transfer trajectory directly in the ROE space, where points have a direct physical meaning (e.g., geometry of the corresponding orbit, one-orbit-minimum cross-track distance) and they move either free of charge (i.e., according to the natural perturbed dynamics - see previous item) or through jumps directly related to the corresponding delta-v consumption.

4. the AVANTI spaceborne navigation system has implemented several countermeasures to robustly operate into a definitely more demanding scenario compared to the one of the PRISMA mission. The target identification task, in fact, has been challenged by frequent and extensive outages of pictures, due to the presence of eclipses and Sun blinding phases (note that BIROS presents only two camera heads). On the other hand, the relative navigation filter could process only few, non-evenly distributed, observations per orbit (i.e., worst-case conditions for a weakly observable problem), while additionally estimating the mean time-derivative of the relative semi-major axis, to catch the strong effect of differential non-conservative orbital perturbations. As a result, in order to improve the overall system robustness, 
the target identification process and the dynamic filtering of the relative motion are handled independently at far range, prior to the filter convergence [21]. And, the luminosity information is only used when it becomes evident that the brightest spot corresponds to the target itself.

5. AVANTI has flight qualified a complete (i.e., self-contained), flexible (i.e, compliant with operational and platform constraints), and computationally light (i.e., running on the same processor already used for the BIRD - Bispectral Infra-Red Detection - satellite in 2001 [22]) spaceborne GNC system. Contrary to the contributions occurred on PRISMA, which made an extensive use of the specific products of the VBS sensor, here only a basic functionality of the star-tracker camera is employed: the capability to extract regions-of-interests pixel areas around each luminous spot exposed (i.e., both stellar and nonstellar objects). As proof of the portability of the AVANTI spaceborne GNC system, one should note that de facto it has been implemented on a small spacecraft platform not specifically designed to perform formation-flying activities.

As a result, AVANTI indeed performed the first rendezvous to noncooperative target object in LEO, moreover in a fully autonomous fashion. The strongly perturbed orbit of BIROS, furthermore creating eclipses which lead to periodic outages of the visibility of the target satellite, constituted a worst-case scenario against which proving the feasibility of the LOS-basedonly approach. These very harsh test conditions, made AVANTI becoming an extremely representative technological demonstration to enable OOS [23.

After this introduction, the paper presents space and ground segments of the AVANTI demonstration (Section 2), focusing on their main elements (i.e., active spacecraft 2.1 and experiment control center 2.3) and on its noncooperative structure 2.2. The central part of the work targets algorithms and implementation of the main modules of the GNC flight software (Section 3), namely: navigation system 3.1, relative orbit guidance and control 3.2, attitude guidance 3.3, and formation safety monitoring unit 3.4 . Afterwards, the results obtained during the flight campaign are presented and discussed (Section 4). Conclusions are drawn in Section 5.

\section{The AVANTI Demonstration}

The AVANTI formation-flying demonstration was one of the secondary scientific objectives of the FireBird mission: a DLR small-scale scientific mission primarily meant for Earth-observation and hot-spot detection [24]. In the frame of FireBird, the BIROS (Bi-Spectral InfraRed Optical System) spacecraft has been inserted into an almost circular, Sun-synchronous 
(local time of ascending node 21:30), $515 \mathrm{~km}$ high orbit with the Polar Satellite Launch Vehicle (PSLV) C-34 launch on the $22^{\text {nd }}$ of June 2016. And formation-flying activities could be performed exploiting the fact that BIROS carried onboard the BEESAT-4 (Berliner Experimental and Educational SATellite) spacecraft, a one-unit CubeSat developed by the Technical University of Berlin [25]. This third-party, independent, experimental activity, in fact, has been used as noncooperative target for the sake of the AVANTI demonstration. BEESAT-4 has been released in-orbit on the $9^{\text {th }}$ of September 2016 by means of a single picosatellite launcher device which provided an equivalent separation delta-v of circa $1.5 \mathrm{~m} / \mathrm{s}$ (see details on the separation strategy design [26, 27] and in-flight events [1]).

\subsection{The BIROS Spacecraft}

Evolved from the ancestor platforms of the DLR small satellites family BIRD and TET-1 [28], the BIROS spacecraft has wet mass of $\approx 140 \mathrm{~kg}$ and an envelope size of $670 \times 580 \times 880 \mathrm{~mm}$ in launch configuration (Fig. 1). Electrical power for the operation of bus and payload is generated by three solar panels (two deployable) delivering a maximum of $220 \mathrm{~W}$ end-of-life. A nickelhydrogen, $250 \mathrm{Wh}$ capacity, battery provides the energy in the eclipse phase or during peak power demands. BIROS power thermal system (PTS) has been sized to support its primary mission goal: all the time not dedicated to take pictures of hot-spots on the Earth surface is spent in an inertial-fixed Sun-pointing mode. Communication is based on an S-band transmission system. It features two receiver/transmitter pairs which can be switched to an omnidirectional low-gain antenna system or to the high-gain antenna. This latter configuration allows achieving the maximum downlink rate (i.e., $2.2 \mathrm{Mbps}$ ) though requiring a special attitude orientation: the high-gain antenna in Nadir pointing.

BIROS is a 3 -axis stabilized satellite and inherits onboard computer (OBC), attitude sensors and actuators gear, and attitude control system (ACS) from the preceding platforms [22, 29]. For absolute positioning, BIROS embarks two, cold redundant, Phoenix GPS receivers [30] whose data are processed by the Onboard Navigation Subsystem (ONS) application. The OBC features: an industrial Power PC 823e processor without floating point support operated at $48 \mathrm{MHz}$ clock rate (66 MIPS of performance), 16 MB SDRAM memory (parity protected), 16 MB SDRAM shadow mirror memory for one bit error detection and correction, and $32 \mathrm{MB}$ of permanent flash memory [22]. The OBC is operated by the BOSS (Basic Operating System Structure) $\mathrm{C}++$ operating system, well suited for real-time and onboard applications.

The propulsion system, instead, is a novelty, as well as a precondition for enabling AVANTI. BIROS is equipped with a Microjet 2000 cold-gas resistojet developed by Aerospace Innovation GmbH and DLR. Since noz- 


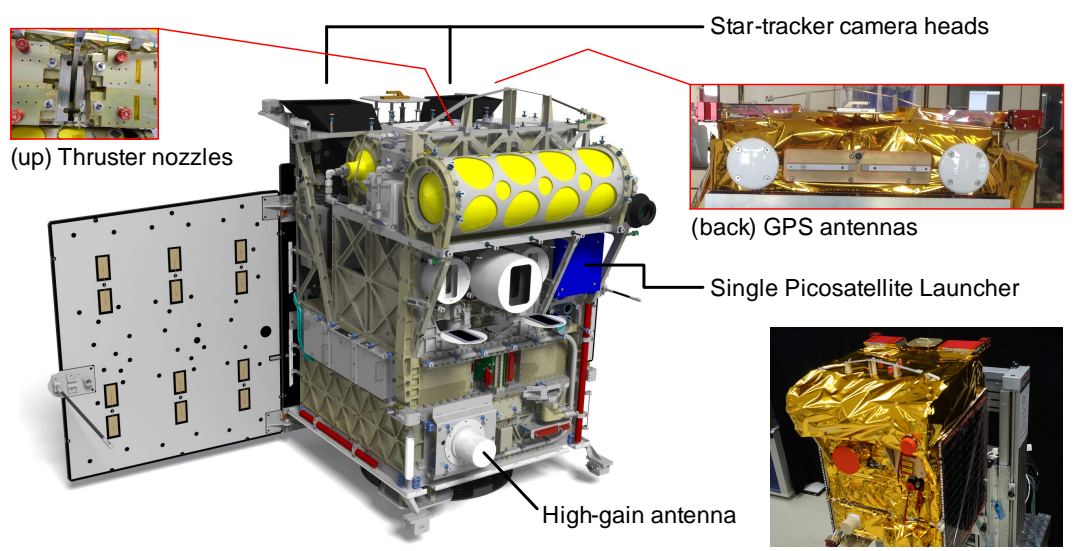

Figure 1: BIROS bus and payload assembly (left); in pre-launch configuration (bottom-right).

zles are activated one at a time, this system generates thrust in a single direction (i.e., one-axis delta-v capability), with a nominal value of $0.1 \mathrm{~N}$ and minimum impulse bit of $1 \mathrm{~s}$ (translating to single velocity increment of approximately $1 \mathrm{~mm} / \mathrm{s}$ ).

Another advance of the BIROS platform is the pre-processing unit (PPU): a supplementary payload computer primarily meant to operate the main payload (i.e., the infrared camera sensor). Relevant for AVANTI, the PPU hosts an experiment-dedicated memory partition (80 MB of space) to store collected pictures and telemetry data.

\subsubsection{Vision-Based sensor}

The primary sensing instrument used within AVANTI is the micro Advanced Stellar Compass ( $\mu \mathrm{ASC})$ star-tracker built by DTU. It features two camera heads (CHUs) and two, cold redundant, digital processing units (DPU) to deliver the two camera-to-inertial-frame quaternions at $4 \mathrm{~Hz}$. In addition, it can export pictures with different compression formats. Given the combination of DPU-OBC serial connection and software extraction of the image, it became impossible to directly process raw-bitmap images. The supported transfer data rate (1-2 KBps), in fact, translated into a picture every 3 to 4 minutes, which was not acceptable to fulfill angles-only relative orbit determination, considering that the number of observations is already strongly limited by the action of eclipses and camera-blinding phases (i.e., orbit geometry constraint). Among the image compression formats offered by the $\mu$ ASC sensor, AVANTI made use of the regions-of-interest (ROIs) one: only the fixed-size area around the brightest luminous spots is kept (without loss of information). Finally, the $\mu \mathrm{ASC}$ sensor implements an electronic shutter to regulate the exposition time. Given the selected compression format, this 
feature is crucial to reduce the centroiding error at close-range distance: the target luminous spot fits better into the ROI-size limitation.

\subsection{Noncooperative Mission Scenario}

The space segment of the AVANTI demonstration is composed by the BIROS and BEESAT-4 spacecraft (Fig. 2). In order to understand what is meant with noncooperative mission scenario, one has to consider the available navigation data sources, for both satellites, with their related accuracies. BIROS's ONS subsystem processes the GPS navigation fixes to provide onboard absolute navigation within $20 \mathrm{~m}$ accuracy. Although BEESAT-4 also embarks a Phoenix GPS receiver (i.e., main payload for the picosatellite [31]), it was not yet commissioned by the time of AVANTI execution. The same occurred to the inter-satellite link: another third-party experimental activity not yet functioning in 2016. As a result, BIROS and BEESAT-4 were not able to communicate during AVANTI flying activities, and no accurate GPS-based information of BEESAT- 4 was available. Alternative data sources to accomplish the absolute orbit determination of the picosatellite could be TLE and radar tracking observations. Nevertheless, both these options are not suitable for mid- to close-range inter-satellite separations and/or in real-time. Standard NORAD (North American Aerospace Defense Command) TLE products, in fact, are typically accurate only to $1-5 \mathrm{~km}$ in the along-track direction, and updated every 3-5 days. A radar-tacking campaign with support of the TIRA (Tracking and Imaging Radar) ground station of the Fraunhofer-Institut für Hochfrequenzphysik und Radartechnik has been performed in October 2016 [32], requiring a minimum inter-satellite distance of $5 \mathrm{~km}$ to distinguish the signals emitted from the two spacecraft. Nevertheless, this campaign could provide a post-facto reference solution for the vision-based relative orbit determination at far-range [32]. Whereas, the only source of observations covering from far- to close-range domains and available in real-time onboard were the pictures taken with the BIROS star-tracker.

\subsection{The Experiment Control Center}

BIROS is operated from the GSOC premises with support of a ground stations network comprising antennas in Weilheim and Neustrelitz (Germany), St. Hubert (Canada), and O'Higgins (Antarctica). During AVANTI, the number and distribution of the supported ground contacts have been tradedoff considering: experiment autonomy vs safety, and experiment needs vs data budget constraints. Given the onboard data handling system of BIROS, only a small part of the AVANTI telemetry (TM) could be kept on the bus OBC and streamed down in real-time during passes. This house-keeping TM contained only the most relevant information to provide a real-time glimpse 


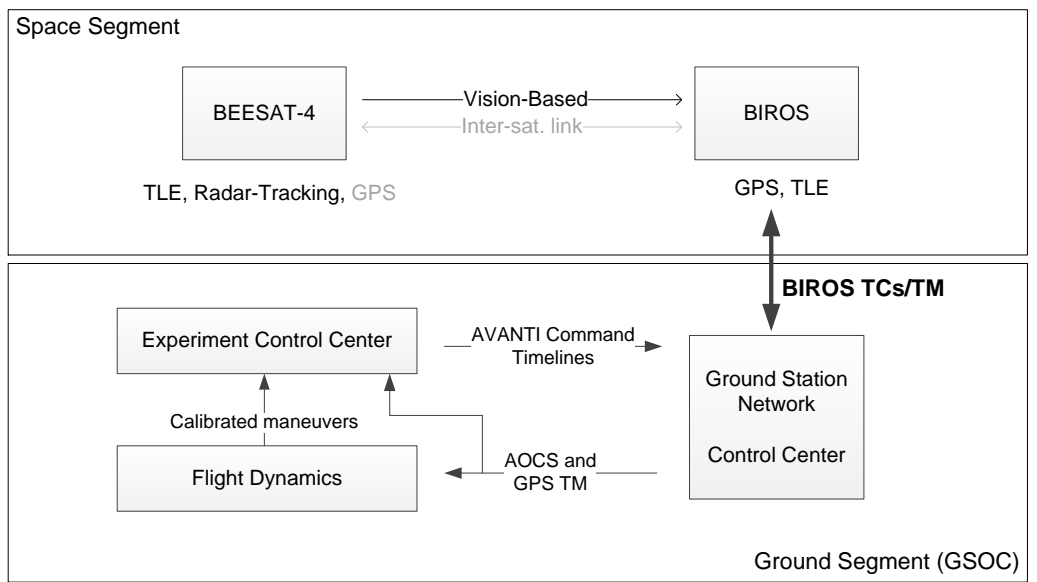

Figure 2: Mission segments of the AVANTI demonstrations: main elements and information flow. Gray items were not available during flight operations.

on the status of the spaceborne system. The remaining data (i.e., extended TM and pictures), necessary to assess the system performance, were stored in an experiment-dedicated memory partition of the PPU board and afterwards accessible on-ground off-line. Therefore, the promptness of any possible reaction of the experimenters' team was driven by the availability on-ground of the data coming from the PPU. Recalling BIROS' communication system, this required the high data-rate (HR) transmission configuration combined with a special attitude orientation (i.e., Earth-pointing mode). HR contacts, however, conflict with the most desirable functioning of the AVANTI GNC: they interrupt the visual-data collection phases (i.e., the target exits the camera field of view), and interfere with the execution of the maneuvers autonomously computed onboard (i.e., single-direction propulsion system, more details later in section 3.2). As a result, taking all these conflicting aspects into account, AVANTI has been operated using a maximum number of $6 \mathrm{up} /$ down plus 1 down-only links every 24 hours. Among these, $4 \mathrm{up} /$ down contacts occurred during office hours, and only 2-to-3 of the down-links, as more evenly distributed as possible, have been configured as HR contacts. This operation concept allowed achieving a compromise between autonomy, data-availability, and responsiveness from-ground, throughout in-flight activities presenting different levels of criticality.

By referring to the bottom part of Fig. 2, the off-line TM of AVANTI, together with GPS-raw data as well stored in the BIROS PPU, have been routinely processed by the experiment control center (ECC), also located in GSOC. Its main activities consisted in: (i) performing the relative precise orbit determination, benefiting from calibrated maneuvers information, to assess spaceborne navigation performance [32] and to re-initialize the onboard safety monitoring tool [15]; (ii) analyzing the extended TM to monitor 
the whole GNC behavior and to detect eventual anomalies; (iii) producing and validating the command timeline that encoded the high-level goals of subsequent phases of the experiment. These activities have been supported by dedicated tools of the AvantiSimulator, a QT-based C++ software environment (also featuring different on-need hardware-in-the-loop capabilities), evolved from the multi-satellite simulator test-bed in use in GSOC to design, integrate, validate, and support spaceborne formation-flying activities in the last decade [33, 34].

\section{The GNC Flight SW}

Figure 3 shows the functional view of the GNC spaceborne system that supported the AVANTI experiment. It is composed by two main modules, respectively named AVANTI and OSM (onboard safety monitoring), which are implemented on the OBC board (two dashed-blocks in Fig. 3). The choice of running these applications on the bus OBC instead of on the more advanced PPU payload computer derived from the following technical (and non-) aspects: (i) to simplify the management of interfaces with respect to the required devices of the platform; (ii) to benefit from the availability of a consolidated system since the early steps of the development of AVANTI (recalling section 2.1, the PPU is itself a technology innovation of BIROS compared to TET-1, hence developed in parallel to other experimental systems). The AVANTI unit features the main relative orbit navigation and control specific functions (in addition to usual TC/TM handling, here not relevant to the discussion). OSM carries out the collision avoidance task. Clearly OSM could have been incorporated in AVANTI, as an additional formation-flying-related sub-module. Nevertheless, the actual strategy has been preferred to achieve a simpler architecture of two separated threads with different priorities and to realize a transparent relationship between tasks and SW entities. This last point fostered clearness between different SW developers and project partners, regarding the critical topic of safety of a multi-satellite mission.

The majority of the interfaces to this GNC system are with the SW layer of the BIROS AOCS. Particularly, it receives absolute translational and rotational states, images from the active CHU, and a feedback of the performed thruster activity. At the same time, AVANTI provides to the AOCS an attitude command and OSM is in charge to forward the first incoming maneuver command. The remaining input interface is with the PTS subsystem which provides temperature data from some core devices to support the attitude guidance reasoning (more details later in paragraph 3.3 of this section). Finally, recalling the aforementioned data budget constraints, extended TM and pictures are forwarded to the experiment partition on the PPU. Note that the thruster control unit is implemented on the PPU, as the 
propulsion system itself is considered as a technological payload and not as an element of the bus. In the sequel algorithms and main implementation aspects of each sub-module are presented.

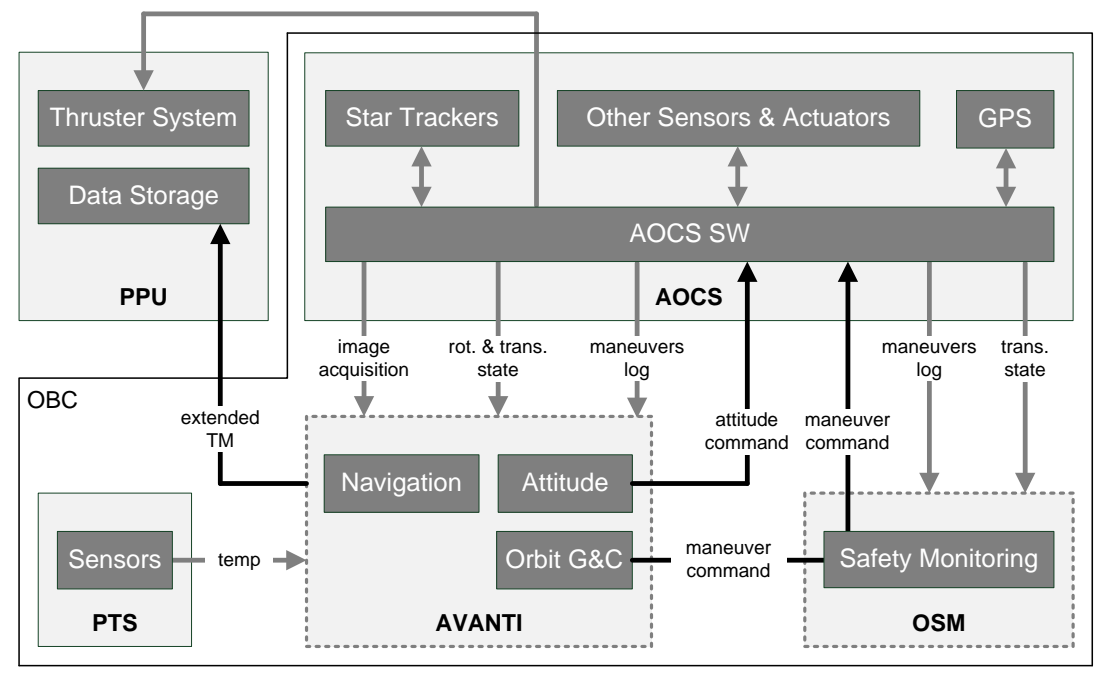

Figure 3: Architecture of the spaceborne GNC SW: functional view and main interfaces with $\mathrm{OBC}$ and PPU.

\subsection{Navigation System}

The onboard navigation system is in charge to firstly identify the target spacecraft out of the pictures collected by the star-tracker, and, subsequently, to continuously estimate the relative state of the formation by processing such LOS observations. To this end, it encompasses the following set of activities: image segmentation, centroiding, stars identification, estimation of the camera attitude, target identification, and dynamic filtering of the angles-only observations. The first sub-tasks basically duplicate some star-tracker regular activities. Nevertheless, they have been retained in the navigation system design to achieve a self-contained angles-only customized GNC system, independent from the specific sensing instrument in use. At the operative level, the main challenge for the target identification task is to achieve a robust and reliable result despite the various luminosity conditions encountered at different separation-ranges and orbit-phases and despite frequent data outages due to eclipse, sensor blinding, and conflicting attitude modes. Data gaps impact also the relative navigation filter: sparse, non-even distributed measurements constitute a worst-case scenario to solve a weakly observable problem. Within AVANTI such issue gets even worse given the need to estimate the effect of the differential aerodynamic drag perturba- 
tion. From the one hand, the frequent small maneuvers, anyway needed to perform the rendezvous, are used to improve the observability property of the problem. From the other hand, being such maneuvers not estimated onboard, the navigation system has to cope with unknown maneuver execution errors. Recent theoretical research is investigating the use of non-linear filtering approaches to improve the maneuver-free angles-only observability property [35] or focuses on the initial relative orbit determination problem [36] to provide accurate initial conditions to the onboard system.

Regarding AVANTI, the algorithms of the spaceborne navigation system evolved from the early design of Ref. [21] to the flight-release form described in Ref. [37]. According to them, the target identification task firstly exploits a kinematic approach based on the fact that the target apparent trajectory differs greatly from the trajectories of other parasite bodies. The target luminosity information is exploited only to perform an integrity check in the case that several candidate clusters are very close to each other, thus presenting an almost equivalent trajectory fitting score. Once that the onboard filter has converged to a valid solution, the target identification task can benefit from such further information to support the first iterations of the kinematic process after each extensive data gap. The onboard relative navigation filter is implemented as an extended Kalman filter which exploits the simple, though accurate, model for the relative motion in near-circular low Earth orbits of Ref. [16]. Accordingly, the relative motion is parametrized by the following set of dimensionless relative orbital elements (ROEs):

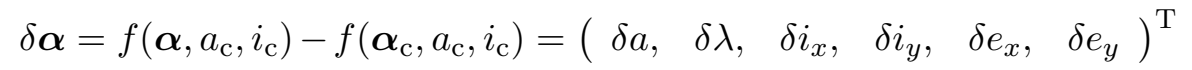

where

$$
f\left(\boldsymbol{\alpha}, a_{\mathrm{c}}, i_{\mathrm{c}}\right)=\left(a / a_{\mathrm{c}}, u+\Omega \cos i_{\mathrm{c}}, i, \Omega \sin i_{\mathrm{c}}, e \cos \omega, e \sin \omega\right)^{\mathrm{T}}
$$

$\boldsymbol{\alpha}=(a, e, i, \Omega, \omega, u)^{\mathrm{T}}$ is the set of classical Keplerian orbital elements, $u$ is the spacecraft mean argument of latitude, and the subscript "c" labels the BIROS satellite. The dimensional estimate state is defined as:

$$
\mathbf{x}=(a \delta \dot{a}, a \delta \boldsymbol{\alpha})^{\mathrm{T}}
$$

with the mean time variation of the relative semi-major axis catching the main effect of time-varying differential aerodynamic drag. With respect to the complete state variable of Ref. [16], the mean time derivative of the relative eccentricity vector terms have been discarded, to keep only the minimum strictly necessary elements, being them reconstructed out of a bearing-only navigation problem. The time update at a new epoch $t$ is then performed through the following state transition matrix:

$$
\mathbf{x}(t)=\mathbf{\Phi}\left(t, t_{0}\right) \mathbf{x}\left(t_{0}\right)=\left[\begin{array}{cc}
\mathbf{I}_{1 \times 1} & \mathbf{O}_{1 \times 6} \\
\tilde{\mathbf{\Phi}}_{\mathrm{d}-\mathrm{drag}}\left(t, t_{0}\right) & \mathbf{\Phi}_{\mathrm{HCW}}\left(t, t_{0}\right)+\mathbf{\Phi}_{\mathrm{J} 2}\left(t, t_{0}\right)
\end{array}\right] \mathbf{x}_{0}
$$


where "HCW" denotes the Hill-Clohessy-Wiltshire contribution, "J2" the mean effects produced by the Earth's oblateness perturbation, and $\tilde{\boldsymbol{\Phi}}_{\mathrm{d} \text {-drag }}$ collects the joint effect of differential aerodynamic drag and $J_{2}$; their functional expressions are given in [16. The measurement update step and the process noise trade-off and settings are respectively described in Ref. [38] and [37].

Regarding the implementation aspects, the whole navigation task (i.e., image processing and relative navigation filter) is executed every $30 \mathrm{~s}$. This feature derives from the image extraction characteristics described in section 2.1.1 and from the computational performances of the onboard processor and leaves no room to implement computational demanding filtering techniques.

\subsection{Relative Orbit Guidance and Control}

The relative orbit guidance and control unit is in charge to compute the impulsive maneuvers' profile to achieve an aimed relative state at a given future time, in a fuel efficient, safe, and feasible manner, that is in compliance with several operational constraints dictated by satellite bus and experiment needs. From a practical point of view, the majority of such constraints translate into user-defined time-intervals in which it is not possible to accomplish any maneuver activity (here referred as to no-control windows). Within AVANTI, this occurs to avoid firing during ground-station contacts scheduled for data dump through the high-gain antenna, to avoid interfering with specific phases of visual data collection, and to guarantee occasionally slots of some hours with no orbit corrections. In addition to these no-control windows, the single-direction thruster system of BIROS imposes further time-constraints to allow slewing the nozzle into the proper direction before firing. Finally, minimum and maximum delta-v magnitudes constraints are directly related to the sizing of the nozzle to provide a $1 \mathrm{~mm} / \mathrm{s}$ minimum control authority (i.e., an experiment design requirement), thruster system functioning, and capability of the reaction wheels to absorb disturbing torques during extended maneuvers.

As for the mathematical formalization of the relative orbit guidance task, it can be seen as a fixed-time reconfiguration problem, where the minimization of the delta-v consumption is sought. Thus, it is a constrained optimization problem where a final state has to be achieved minimizing a cost that is function of number, magnitude, direction, and execution time of the maneuvers. Furthermore, recalling the harsh noncooperative mission scenario described in section 2.2 , the reconfiguration transfer trajectory shall preserve a certain minimum cross-track separation margin with respect to BEESAT-4, to guarantee an intrinsic collision free approach (i.e., passivesafety).

Being part of an autonomous onboard system, this module has been 
designed to fulfill determinism and low computational load. The first aspect, in fact, concerns the capability to find in most cases a viable solution. The latter is a hard requirement deriving from the characteristics of the BIROS onboard computer (see section 2.1). Keeping in mind the complexity of the problem and the aforementioned design drivers, the originality of the adopted solving approach consists in splitting it into more treatable, sequentially connected, sub-tasks namely: constraints handling, trajectory design through way-points in the ROE space, and consequent placement in the time-plan of the required impulsive maneuvers. For simplicity, time constraints are solved firstly to restrict the solution's domain. The remaining two tasks can be tackled sequentially thanks to the following peculiarities of the ROE framework: (i) the availability of a simple but accurate and complete closed-form state transition matrix for the perturbed relative motion; (ii) the geometrical meaning of each component of the ROE state; (iii) the possibility to easily and synthetically express the passive-safety property of a relative orbit as function of the ROE state components [15, 39]; (iv) the functional structure of the relations between instantaneous variation of velocity in the local radial-tangential-normal (RTN) orbital frame and consequent effect on the ROE components [40]. All these features together allow transforming a time-dependent optimal control problem into a geometrical minimum-path problem in the ROE space, provided that the maneuvers scheduled to achieve each intermediate way-point are computed pursuing the same optimality criterion of the way-points planning step.

Description and detailed development of the early design of the core maneuver planning algorithm is presented in Ref. [20]. An orbit reconfiguration is expressed as the evolution of the current state $\mathbf{x}_{0}$ to an aimed one at a future final time $\mathbf{x}_{F}$ as a sequence of intermediate way-points $\mathbf{x}_{i}$ to be reached at some times $t_{i}$ (i.e., the end-times of the series of permissible time-intervals out of the solution of the scheduling initial task):

$$
\mathbf{x}_{F}=\boldsymbol{\Phi}\left(t_{F}, t_{0}\right) \mathbf{x}_{0}+\boldsymbol{\Phi}\left(t_{F}, t_{1}\right) \Delta \mathbf{x}_{1}+\cdots+\boldsymbol{\Phi}\left(t_{F}, t_{m}\right) \Delta \mathbf{x}_{m}
$$

where the discontinuities $\Delta \mathbf{x}_{i}$ are included on top to the natural perturbed dynamics to introduce the effect of some (so far undefined) maneuvers. Differently from its earlier development based on the state transition matrix of Eq. (6) of Ref. [20, the final release of the algorithm employed during AVANTI exploits the following more complete expression (note that here the components are permuted to highlight that the relative eccentricity vector 
is decoupled):

$$
\begin{aligned}
& \mathbf{\Phi}\left(t, t_{0}\right)= \\
& {\left[\begin{array}{ccccccc}
1 & 0 & 0 & 0 & 0 & 0 & 0 \\
\left(t-t_{0}\right) & 1 & 0 & 0 & 0 & 0 & 0 \\
0.5\left(\nu+\mu_{A}\right)\left(t-t_{0}\right)^{2} & \left(\nu+\mu_{A}\right)\left(t-t_{0}\right) & 1 & \mu_{I}\left(t-t_{0}\right) & 0 & 0 & 0 \\
0 & 0 & 0 & 1 & 0 & 0 & 0 \\
0.5 \lambda_{A}\left(t-t_{0}\right)^{2} & \lambda_{A}\left(t-t_{0}\right) & 0 & \lambda_{I}\left(t-t_{0}\right) & 1 & 0 & 0 \\
0 & 0 & 0 & 0 & 0 & \cos \left(\dot{\varphi}\left(t-t_{0}\right)\right) & -\sin \left(\dot{\varphi}\left(t-t_{0}\right)\right) \\
0 & 0 & 0 & 0 & 0 & \sin \left(\dot{\varphi}\left(t-t_{0}\right)\right) & \cos \left(\dot{\varphi}\left(t-t_{0}\right)\right)
\end{array}\right]} \\
& \nu=-(3 / 2) n \quad \quad \mu_{A}=-(21 / 4) n \gamma\left(3 \cos ^{2} i-1\right)(\eta+1) \quad \mu_{I}=-(3 / 2) n \gamma \sin (2 i)(3 \eta+4) \\
& \dot{\varphi}=+(3 / 2) n \gamma\left(5 \cos ^{2} i-1\right) \quad \lambda_{A}=+(21 / 4) n \gamma \sin (2 i) \quad \lambda_{I}=3 n \gamma \sin ^{2} i \\
& n=\sqrt{\mu_{\oplus} / a^{3}} \quad \gamma=\frac{J_{2}}{2} \frac{R_{\oplus}^{2}}{a^{2} \eta^{4}} \quad \eta=\sqrt{1-e^{2}}
\end{aligned}
$$

Eq. (6), in fact, includes also the changes in both relative mean longitude and relative inclination vector due to the $J_{2}$ and differential aerodynamic drag combined perturbations for drifting relative orbits as in [16]. In addition, no linearization is made to the rotation over time of the relative eccentricity vector caused by the $J_{2}$ term as in [41]. This last aspect becomes relevant in the guidance problem, where the propagation time can span over days against the 30 seconds step of the navigation filter. Moreover, since subblock of Eq. (6) satisfies the property:

$$
\boldsymbol{\Phi}\left(t_{j}, t_{i}\right) \cdot \mathbf{\Phi}\left(t_{i}, t_{k}\right)=\mathbf{\Phi}\left(t_{j}, t_{k}\right)
$$

the approximation of Eq. (14) of Ref. [20] is no more required.

The cumulative delta- $\mathrm{v}$ cost associated to the $\mathbf{x}_{0} \rightarrow \mathbf{x}_{F}$ reconfiguration can be expressed by the following quadratic function of the ROE corrections not related to the natural dynamics (i.e., the $m \Delta \mathbf{x}_{i}$ jumps):

$$
J_{\text {plan }}=\sum_{i=1}^{m}(\|a \Delta \delta \mathbf{i}\|)_{i}^{2}+\sum_{i=1}^{m}(a \Delta \delta a)_{i}^{2}+\sum_{i=1}^{m}(a \Delta \delta \lambda)_{i}^{2}+\sum_{i=1}^{m}(\|a \Delta \delta \mathbf{e}\|)_{i}^{2}
$$

where $a \delta \mathbf{e}$ and $a \delta \mathbf{i}$ are respectively the relative eccentricity and inclination vectors.

In the ROE space, Eq. (8) is a metric of distance measuring the length of the segments obtained with additional cost (i.e., delta-v). Thus, minimizing $J_{\text {plan }}$ means connecting $\mathbf{x}_{0}$ and $\mathbf{x}_{F}$ in the cheapest possible way (i.e., minimum-path problem), that is reducing the necessary fuel consumption. Note that this approach naturally includes the trade-off between $|\Delta \delta a|$ and $|\Delta \delta \lambda|$ corrections, thanks to their relationship embedded in $\boldsymbol{\Phi}$. Or equivalently, provided that the time to carry out a reconfiguration is long enough, the discontinuity term associated to the mean relative longitude change (well-known achievable only with expensive radial burns), tends to zero, as it is absorbed in the definitely smaller relative semi-major axis change to establish the proper drift acting over the whole available time elapse. The 
minimization problem of Eqs. (5) and (8) is convex and presents a global minimum obtainable fully analytically. The optimal values of the ROE discontinuities are obtained solving the linear system expressing the optimality necessary conditions for the stationary point of Eq. (8). The closed-form analytical solution of the so upgraded maneuver planning problem is presented in Appendix 5.1 to this paper.

Once given the delta-v optimal changes of ROEs, the last guidance step computes the effective impulsive maneuvers, allocated only in the permissible (i.e., constraint-free) time-intervals, required to achieve each intermediate way-point at its proper time. Such delta-v minimum local sub-problems demand only tangential and normal orbit corrections. Referring to Fig. (8) of Ref. [40], among the possible double/triple-impulse solution schemes able to satisfy the whole set of ROE end-conditions at a given time, AVANTI implements Eq. (8) of Ref. [40] for the out-of-plane correction and the option \#12 (see also Table 2 of Ref. [40]) for the in-plane reconfiguration (more details in Appendix 5.2. This latter foresees a sequence of three tangential impulses placed at half orbital period multiples of the mean argument of latitude corresponding to the phase angle of the aimed relative eccentricity vector change. Its choice is motivated by the level of determinism and predictability of such a solution, which guarantees the existence of at least a (delta-v minimum) analytical solution given a 2-orbit period shortest possible time for allocating all three maneuvers. More details concerning the logic of allocation of the required maneuvers in the admissible time intervals are provided in Section 6.3 of Ref. [20]. Note that the selected burning scheme is straightforward compliant with the maneuvers' spacing time-constraint to slew the single-direction thrusters' system.

Furthermore, the solution obtained through the aforementioned relative orbit guidance algorithm intrinsically guarantees that if a passivelysafe final state is reached from an initial one with similar relative eccentricity/inclination phasing characteristics, then the transfer trajectory is passively-safe during its whole duration, provided that the relative semimajor axis remains small enough (i.e., the reconfiguration takes place over a long enough time horizon), even if the maneuver plan is interrupted at any time prior to its completion (see Section 5 of Ref. [20]).

From an operational point of view, this relative orbit guidance algorithm constitutes the kernel of the maneuver planner unit of the AVANTI GNC module. This receives via telecommand from ground the high-level instruction of reaching a certain relative state at a given future time and produces a specific maneuver schedule depending on the selected operative mode. The available options are summarized in Table1, categorized by main characteristics and way to handle the no-control windows time-constraints. Details regarding min delta- $v$ and max observability modes are provided in Section 2.1 of Ref. 20]. Accordingly, the first prescribes a pure open-loop guidance, not appropriate to obtain an accurate control on the state, but 
Table 1: Maneuver planner operative modes.

\begin{tabular}{|c|c|c|c|}
\hline N & Mode & Characteristics & Constraint handling \\
\hline 1 & Min delta- $\mathrm{v}$ & $\begin{array}{l}\text { absolute minimum } \\
\text { delta-v cost, up to } 4 \\
\text { burns } \forall \text { plan }\end{array}$ & $\begin{array}{l}\text { maneuvers spread over } \\
\text { whole plan horizon, } \\
\text { burns only in permis- } \\
\text { sible intervals }\end{array}$ \\
\hline 2 & Max observability & $\begin{array}{l}\text { maneuvers activity } \\
\text { distribution, up to } \\
4 \times m \text { burns } \forall \text { plan, } \\
\text { autonomous re-plan }\end{array}$ & $\begin{array}{l}\text { permissible intervals } \geq \\
2.5 \text { orbits, } 1 \text { maneuver } \\
\text { group } \forall \text { free time-slot }\end{array}$ \\
\hline 3 & Station-keeping & $\begin{array}{l}\text { up to } 4 \text { burns } \forall \text { duty- } \\
\text { cycle, autonomous re- } \\
\text { plan }\end{array}$ & $\begin{array}{l}\text { duty-cycle } \geq 2.5 \text { or- } \\
\text { bits, duty-cycle ex- } \\
\text { tended if overlapping } \\
\text { to no-maneuver slot }\end{array}$ \\
\hline
\end{tabular}

preferred during semi-autonomous mission phases, that is when a certain level of supervision is performed from ground, regarding performance of the navigation system and occurrence of the thruster activation. The second operative mode exploits more frequent maneuvers to improve the observability property of the angles-only vision-based relative navigation [42. After the achievement of every intermediate way-point, the remaining plan to the final target is autonomously updated on the current navigation solution (i.e., from $t_{i}$ to $t_{F}$ ), thus closing the feed-back control loop. As a result, the userdefined no-control windows, in addition to their primary purpose, serve also the scope to allow the user managing the distribution of the maneuvering activity. Note that the use of solely tangential and normal impulsive maneuvers constitutes a synergy of the developed GNC design: from the one hand they are required to minimize the delta-v, from the other one they are more effective in improving the angles-only navigation observability [38]. The last operative mode is named station-keeping, since it aims at maintaining an almost-bounded relative orbit over extended periods of time. It is implemented as the indefinite series of reconfigurations from the current state to the target one, where each reconfiguration is accomplished by a group of maneuvers within the duration of a prescribed duty-cycle. As maneuvers are computed to enforce the aimed state at a given time, and as they are groupwise updated once reached the duty-cycle due time (i.e., at re-plan time), this mode realizes a coarse control, whose accuracy mainly depends from the duty-cycle duration, coherently with its reiterated open-loop structure.

The linking between navigation system and G\&C determines how the overall control loop is closed. Although G\&C interacts with all the main tasks of the AVANTI-OSM flight SW, Fig. 4 focuses on its communication with relative navigation and formation safety monitoring threads. The G\&C 


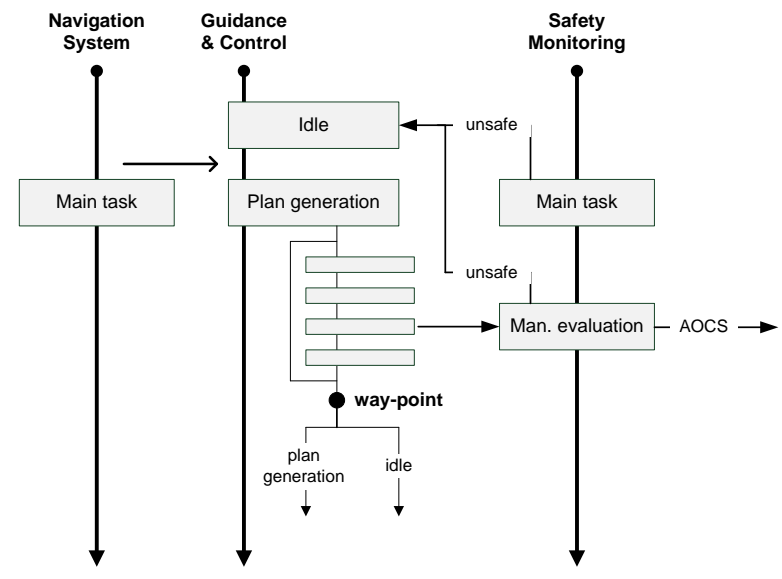

Figure 4: G\&C task and its interrelations with other functions of the AVANTI and OSM SW modules.

is implemented with the typical receding finite-time horizon of the model predictive control (MPC). The prediction horizon equals the time from the plan update moment (i.e., plan generation state) to the aimed final time of the whole rendezvous horizon. Whereas the control horizon is the time to achieve the first incoming intermediate way-point. Such communication architecture results from the trade-off between responsiveness of the control and achievable overall accuracy: a prompter feed-back control can provide better accuracy but can also lead to a non-strictly required delta-v waste. For noncooperative rendezvous using only a monocular sensor system, the challenge is to step-wise refine both navigation and control solutions, despite a weakly observable navigation problem and unknown maneuver execution errors. Furthermore, updating the plan only after the completion of each maneuvers' group, allows keeping the computational load extremely low. Maneuvers are simply recomputed through the usual, purely analytical, locally delta-v optimal, solution scheme. As a result, the implemented architecture exploits typical benefits of MPC like the capability to enforce constraints on input (i.e., time constraints on the time of the maneuvers) and outputs (i.e., end-condition and passive safety), and to optimize a performance index (i.e., fuel consumption). At the same time, it mitigates the MPC drawbacks of a larger computational load of classical (linear) control methods, having reduced the optimal planning problem to the solution of a linear convex problem in the ROE space.

To conclude, the safety, in the sense of collision avoidance, of the so obtained controlled trajectory is continuously monitored by the OSM module (see Section 3.4), which therefore accomplishes a kind of external supervision with the steady authority to clear an ongoing maneuver plan (i.e., forcing 
Table 2: Attitude modes definitions with respect to two fixed or timevarying directions in the RTN orbital frame. Superscripts $c$ and sat respectively denote the camera and satellite body-fixed reference frames.

\begin{tabular}{ccc} 
Mode & First direction & Second direction \\
\hline COM & $\mathbf{z}^{\mathrm{c}} \| \mathbf{y}^{\mathrm{RTN}}$ (far-range $)$ & $\mathbf{y}^{\mathrm{c}} \| \mathbf{R}_{\mathbf{z}^{\mathrm{c}}}(\alpha)\left(\left(\mathbf{I}-\left[\mathbf{z}^{\mathrm{c}} \times\right] /\left(\mathbf{z}^{\mathrm{c}} \cdot \mathbf{z}^{\mathrm{c}}\right)\right) \cdot \mp \mathbf{x}^{\mathrm{RTN}}\right)$ \\
& $\mathbf{z}^{\mathrm{c}} \| \mathbf{u}^{\mathrm{RTN}}($ mid/close-range $)$ & $\min \angle\left(-\mathbf{z}^{\mathrm{sat}}, \mathbf{I}-\left[\mathbf{z}^{\mathrm{c}} \times\right] /\left(\mathbf{z}^{\mathrm{c}} \cdot \mathbf{s}^{\mathrm{RTN}}\right)\right)$, light \\
& & $\min \angle\left(-\mathbf{z}^{\mathrm{sat}}, \mathbf{I}-\left[\mathbf{z}^{\mathrm{c}} \times\right] /\left(\mathbf{z}^{\mathrm{c}} \cdot \mathbf{x}^{\mathrm{RTN}}\right)\right)$, shadow \\
\hline CDM & $\mathbf{z}^{\mathrm{sat}} \|-\mathbf{s}^{\mathrm{RTN}}$ & $\min \angle\left(\mathbf{y}^{\mathrm{sat}}, \mathbf{x}^{\mathrm{RTN}}\right)$, light \\
& $\mathbf{z}^{\mathrm{sat}} \|-\mathbf{x}^{\mathrm{RTN}}$ & $\min \angle\left(\mathbf{x}^{\mathrm{sat}}, \mathbf{x}^{\mathrm{RTN}}\right)$, shadow \\
\hline EPM & $\mathbf{z}^{\text {sat }} \|-\mathbf{x}^{\mathrm{RTN}}$ & $\mathbf{y}^{\mathrm{sat}} \|-\mathbf{z}^{\mathrm{RTN}}$ \\
\hline TFM & $\mathbf{z}^{\text {thr }} \|-\delta \mathbf{v}^{\mathrm{RTN}}$ & $\min \angle\left(\mathbf{z}^{\mathrm{sat}}, \mathbf{x}^{\mathrm{RTN}}\right)$
\end{tabular}

Note: $\mp$ respectively for CHU0 and CHU1; $\alpha$ rotation angle provided via telecommand.

the entrance to "Idle"). Again referring to Fig. 4, the safety monitoring thread is additionally directly questioned by the planner prior to dispatch a new maneuver command. Only if the post-maneuver trajectory is evaluated as safe, the maneuver command is actually forwarded to the AOCS system.

\subsection{Attitude Guidance}

The attitude guidance unit of the AVANTI SW features the selection of the best-suited attitude mode and, in some cases, computes the reference time-varying pointing profile to be tracked. Functionally, it bridges the rendezvous-specific navigation and control modules of AVANTI to the attitude control task performed by the BIROS ACS system. Its implementation has been in effect required to cope with the high level of autonomy of the onboard maneuver planner and to satisfy the visual-tracking need of keeping the target satellite in the narrow field of view of the camera sensor at close-range (especially considering the passively safe spiraling approach).

The attitude modes employed within this rendezvous demonstration are listed in Table 2 the first two have been expressively conceived to support AVANTI, whereas the remaining ones are standard options of the BIROS attitude system. In Table 2 these modes are defined through two target directions in the RTN frame, depending on orbit (i.e., light and eclipse) and experiment (i.e., inter-satellite range) phases.

The Client Observation Mode (COM) is the peculiar orientation devoted to images data collection. To this aim, the boresight $\mathbf{z}^{\mathbf{c}}$ of the active camera head of the star-tracker is directed to a prescribed direction: the local flight direction (i.e., $+\mathrm{T}$ given that BEESAT-4 leads the formation), or the estimated LOS to the target satellite $\mathbf{u}^{\mathrm{RTN}}$. The attitude definition is completed by a rotation of the camera frame with respect to the boresight axis, in order to take into account other aspects, like power budget and GPS antenna 
visibility pattern, which might become critical during certain COM phases. Referring to Table 2, the first possibility is to command a constant rotation angle $\alpha$ to achieve a compromise respectively between the Sun to the solar panels, and the GPS antenna to Zenith angles. The second option, instead, fosters the power budget aspect, seeking to minimize the angle of the Sun to the normal to panel while in light. By contrast, during eclipses, the satellite z-axis is rotated 180 degrees away, to avoid pointing the GPS antenna to Nadir. As a result, every orbit BIROS rotates to re-orient its panel w.r.t. the Sun and performs two slews, entering and leaving the shadow region, while keeping the camera sensor towards the target s/c. De facto, so defined COM implements a family of time-varying orientations obtainable by selecting: (i) the camera head, (ii) first direction behavior, (iii) second direction preference (with additional tunable parameters).
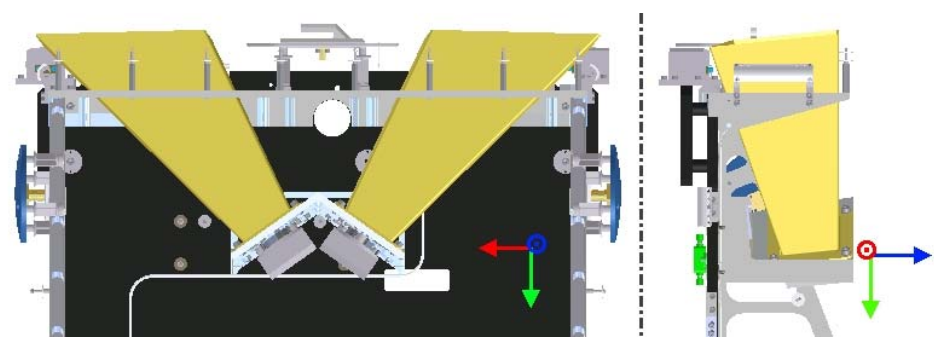

Figure 5: Arrangement of the star-tracker camera heads on the BIROS bus (picture taken from the TET-1 twin platform). The arrows recall the body-fixed spacecraft sat reference frame. CHU0 presents boresight (i.e., $\mathbf{z}^{\mathrm{c}}$ ) directed in $+\mathbf{x}^{\mathrm{sat}} /-\mathbf{y}^{\mathrm{sat}}$; CHU1 in $-\mathbf{x}^{\mathrm{sat}} /-\mathbf{y}^{\mathrm{sat}}$.
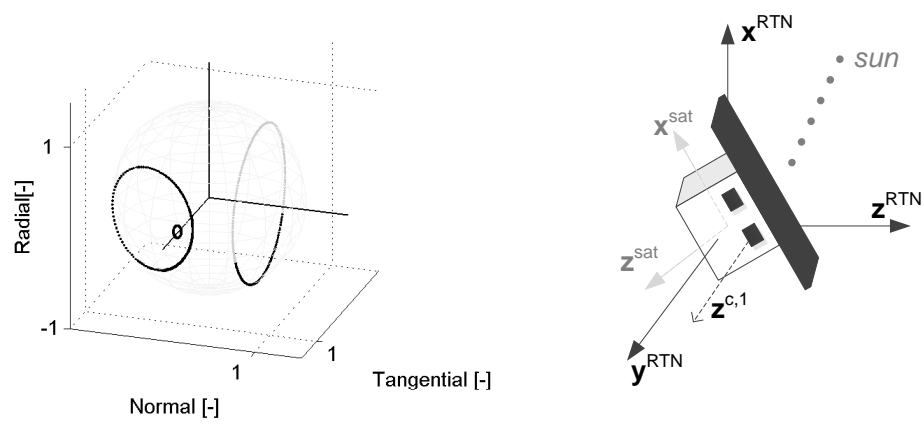

Figure 6: Left: LOS and Sun tracks on the unit-sphere centered on the RTN frame. Right: sketch of BIROS in a COM-like attitude.

The Cool-Down Mode (CDM) is a situation-specific attitude mode additionally introduced to compensate some thermal side-effects of lasting persistence in COM. As shown in Fig. 5, both camera heads present the bore- 
sight mainly directed along the $-\mathbf{y}^{\text {sat }}$ satellite axis. Figure 6-left, instead, presents the LOS and Sun tracks on the unit-sphere centered on the RTN frame for the mission orbit. The LOS depicts a path around the positive flight direction. Though it remains tight at far/middle-range, already at $\approx 120 \mathrm{~m}$ of mean along-track separation it occupies a substantial part of the hemisphere. On the other hand, the Sun describes a circle around the normal to the orbital plane (clockwise); darker dots mark the portion of path in eclipse. Joining scenario and star-tracker arrangement information, Fig. 6 right presents a simple sketch of the resulting typical COM attitude. This is practical to visualize how AVANTI misuses the BIROS platform, which has been designed to spend all the time not dedicated to take pictures of hot-spots on the Earth surface in an inertial-fixed Sun-pointing mode. In COM, firstly, the Sun incidence angle is not that favorable, motivating the exploitation of the rotation around the boresight axis to mitigate power budget issues. Secondly, by keeping the camera directed towards BEESAT-4, BIROS receives the Sun in the radiator once per orbit before the entrance in eclipse, which is exactly when the illumination conditions are the most appropriate to image the target. To avoid causing any damage to the spacecraft, CDM can be used on need: its goal is maximizing the heat dissipation to quickly lower the satellite temperature, while recharging the spacecraft batteries as outlined in Table 2 .

The Earth Pointing Mode (EPM) is the standard orientation required to point the primary science instrument of BIROS to Nadir. Within AVANTI, it is selected during those ground-contacts in which the high-gain antenna is used to stream down data at a greater data-rate (i.e., HR contacts: see experiment data budget and related operations concept in Section 2.3.

The last item of Table 2 is the Thruster Firing Mode (TFM), which is the attitude used to perform orbit correction maneuvers. To this end, the nozzle in use by AVANTI is pointed in the direction required to obtain the delta-v prescribed by the onboard maneuver planner. With the remaining degree of freedom, the satellite is rotated to minimize the angle between GPS antennas and Zenith, to guarantee visibility of as many as possible GPS satellites.

Table 3: Conditions to undertake the autonomous mode transitions of Fig. 7

\begin{tabular}{llcc} 
Transition & Conditions & & Further parameters \\
\hline $\mathrm{COM} \leftrightarrow \mathrm{CDM}$ & $\begin{array}{l}\text { Temperature-based } \\
\text { lance disabled } \\
\mathrm{COM} \leftrightarrow \mathrm{CDM}\end{array}$ & $\begin{array}{l}\text { Temperature-based } \\
\text { lance enabled }\end{array}$ & surveil- \\
& {$\left[u_{\text {start }}, u_{\text {end }}\right],\left[T_{\text {min }}, T_{\text {max }}\right]$} \\
\hline $\mathrm{C}^{*} \mathrm{M} \leftrightarrow \mathrm{TFM}$ & $\begin{array}{l}\text { maneuver or maneuver con- } \\
\text { nected to ground-contact }\end{array}$ & $\Delta t_{\text {slew }}, \Delta t_{\text {post-man }}$ \\
& ground-contact & $\Delta t_{\text {slew }}$
\end{tabular}




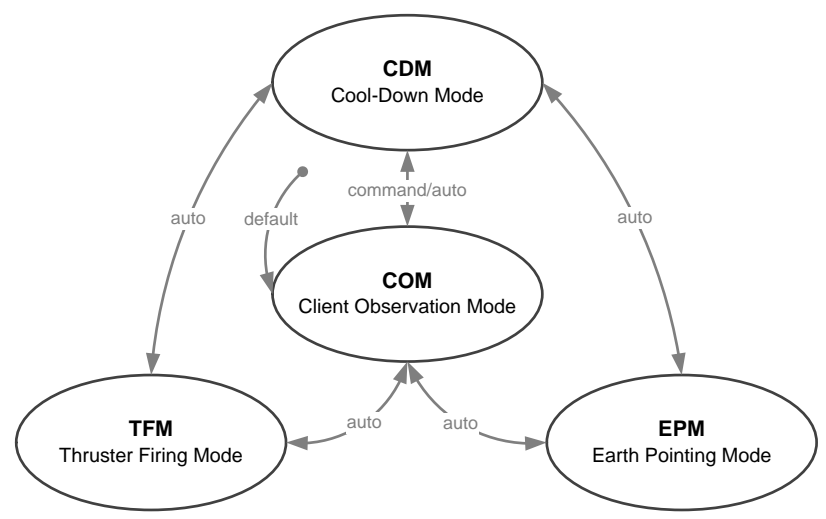

Figure 7: Attitude modes architecture and transitions.

Regarding the flight SW implementation, the aforementioned attitude modes are related to each other according to the graph of Fig. 7. The transitions linking the $\mathrm{C}^{*} \mathrm{M}$, TFM, EPM elements occur fully autonomously onboard, obeying to simple selection principles (see Table 3). As a default, during AVANTI BIROS remains in COM, to satisfy the need of the navigation system of collecting as many as possible observations. TFM is entered, with a proper advance time to complete the slew (i.e., $\Delta t_{\text {slew }}$ ), when a planned maneuver is approaching, or when a maneuver is almost overlapping with a high-rate ground-contact. This rule establishes a preference hierarchy between TFM and EPM inherited from the preliminary s/c design that foresaw the nozzles almost aligned to the $-\mathbf{y}^{\text {sat }}$ direction[2], and thus offering the synergy of the high-gain antenna pointing to Nadir during all maneuvers prescribed by the planner (confined in the T-N plane). In parallel to this option, the user can anyway decouple TFM from EPM simply by wrapping a scheduled ground-contact within a no-control window. Finally, $\mathrm{EPM}$ is entered if an isolated ground-contact is forthcoming. As soon as the main activities requiring either TFM or EPM modes are completed, the onboard guidance mode selects the return to $\mathrm{C}^{*} \mathrm{M}$.

While in COM, the settings that characterize each possible profile of this family of orientations are kept persistently. The user can change the type of observation profile only via telecommand, since this action reflects an experiment decision, rather than an autonomous logic. The interaction between COM and CDM, finally, deserves a special consideration since it realizes the thermal issues mitigation strategy: CDM is required to prevent damages due to high temperatures, but it determines an interruption of the target visual-tracking. The most basic (and less flexible) approach is to handle the $\mathrm{COM} \leftrightarrow \mathrm{CDM}$ transition via command from ground. Much more practical and flexible approaches consist in either defining a portion of orbit (i.e., mean argument of latitude comprised in the $\left[u_{\text {start }}, u_{\text {end }}\right]$ interval) to 
spend in CDM, or exploiting a temperature-based surveillance to detect onboard the need of entering CDM. This latter implements an hysteresis loop on the temperature values of some meaningful devices (e.g., batteries and PPU platforms), where minimum and maximum thresholds are tunable via telecommand. As a result, while BIROS is accumulating heat, the navigation system benefits of the full observations' arc. During cool-down phases, instead, the $u_{\text {start }}$ and $u_{\text {end }}$ parameters balance the trade-off between time required to reach $T_{\min }$ and quantity of measurements' loss per each orbit.

\subsection{Safety Monitoring}

The task of the OSM module is to supervise the safety (in the sense of collision avoidance) of the BEESAT-4-BIROS formation, in real-time and without using the relative orbit solution estimated by the AVANTI SW unit. To this end, OSM exploits a reference relative trajectory which is obtained propagating in time an initial relative state provided from ground (i.e., the best estimation of the relative state at a certain time obtained from the on-ground reprocessing of the latest available telemetry). Within the space segment, in fact, this reference trajectory is regarded as the best available knowledge of the true relative state.

As detailed in Ref. [15], the noncooperative scenario of the AVANTI experiment required to develop a safety concept which does not rely on the continuous availability of any picosatellite tracking data. It is mainly based on the use of passively safe relative trajectories and it is then completed by the following additional safety measures: an onboard preventive action and a long-term reaction. In this framework, OSM implements the onboard functionality, by forwarding to the AOCS a maneuver command generated by the AVANTI unit only if the post-maneuver relative orbit is considered to remain safe at least during a certain amount of hours (tunable via TC) following the evaluation time. On the contrary, the long-term reaction is represented by any required orbit correction maneuver assessed on-ground, based on the analysis of the retrieved telemetry, and generated either through new TCs for AVANTI or directly commanded to BIROS. Note that the wording long-term is used in agreement with the delay to actually retrieve the data from the PPU, considering the experiment operations constraints explained in section 2.3. Hence, this latter countermeasure is meant for contingency situations.

A formation is evaluated as safe if the relative trajectory cannot lead to any collision, within a certain amount of subsequent hours, despite the uncertainty in relative navigation knowledge, especially in the along-track direction. To this end, a certain margin is required between the origin of the RTN frame (i.e., BIROS) and the minimum distance of the one-orbit relative-trajectory projected on the radial-normal $(\mathrm{RN})$ plane (i.e., minimum displacement between the satellites in the cross-track plane). This feature 
is defined as:

$$
\delta r_{\mathrm{RN}}^{\min }=\min _{u \in[0,2 \pi)} \sqrt{(a \delta i \sin (u-\theta))^{2}+(a \delta a-a \delta e \cos (u-\theta-\phi))^{2}}
$$

where $\phi=\varphi-\theta$ is the phase between relative eccentricity and inclination vectors, being $\varphi$ and $\theta$ respectively perigee and ascending node of the relative orbit (i.e., the phase elements of relative eccentricity and inclination vectors in polar notation, see Eq. (3) of Ref. [16]). Note that the function $\delta r_{\mathrm{RN}}^{\min }$ maps the instantaneous relative state $\mathbf{x}(t)$ of Eq. (3) into the corresponding oneorbit minimum $\mathrm{RN}$ distance pictured at time $t$, thus expressing an intrinsic conservative information (i.e., the target might transit through such point in a time comprised in $[t, t+T)$, being $T$ the BIROS orbital period). Thus, the numerical value of $\delta r_{\mathrm{RN}}^{\min }$ is directly related to the magnitude of the relative semi-major axis and to the geometry of the relative orbit, and, with the passing of the time, it varies smoothly due to the action of the drift and of the presence of orbit perturbations according to Eq. (6). Maneuvers, on the contrary, can produce sudden variations in $\delta r_{\mathrm{RN}}^{\mathrm{min}}$. That is why OSM verifies the post-maneuver value of the minimum RN distance, before approving any command generated by AVANTI.

The strength of the main algorithm of OSM is to compute $\delta r_{\mathrm{RN}}^{\min }$ through the explicit and general (i.e., accounting for the contributions of all ROEs) expression of Eq. (12) of Ref. 15. First, it provides a result without approximation errors at a very low computational cost. Second, such explicit formulation enables the use of the unscented transformation (UT) to map the uncertainty distributions of knowledge of the initial state and typical maneuver execution errors into the resulting uncertainty distribution of the minimum RN distance. The UT framework, in fact, offers a simple mechanism to compute the first two moments of a distribution after a nonlinear transformation, achieving projected mean and covariance correct to the second order [43]. It does not require the computation of the partial derivatives of Eq. (12) of Ref. 15, and performs few computations to produce a result (i.e., only 11 sigma points are needed given dimension and structure of the variables' domain). More details are discussed in [15].

The criterion to state the safety of a relative motion is expressed as:

$$
\delta \bar{r}_{\mathrm{RN}}^{\min }-3 \sigma_{\delta r_{\mathrm{RN}} \min }>M
$$

were the symbols $\left(\bar{\bullet}, \sigma_{\bullet}\right)$ identify expected value and standard deviation of the minimum $\mathrm{RN}$ distance distribution, regarded as Gaussian, having represented the uncertainties of $\mathbf{x}\left(t_{0}\right)$ and executed delta-vs Gaussian as well. $M$ is a safety margin, representable as a circle of radius $M$ around the origin of the R-N plane, accounting for: (i) the volume of BIROS; (ii) the maximum approximation error introduced by the UT transformation; (iii) numerical errors in the computation of the $z^{*}$ roots of Eq. (12) of Ref. 15 on a processor not supporting the floating point representation. 
Table 4: Autonomous rendezvous activities.

\begin{tabular}{lccccccc} 
Range & $\begin{array}{c}\text { Start } \\
{[\mathrm{UTC}]}\end{array}$ & $\begin{array}{c}\text { Duration } \\
{[\text { days }]}\end{array}$ & $\begin{array}{c}a \delta \lambda_{0} \\
{[\mathrm{~m}]}\end{array}$ & $\begin{array}{c}a \delta \lambda_{F} \\
{[\mathrm{~m}]}\end{array}$ & $\begin{array}{c}a \delta e_{F} \\
{[\mathrm{~m}]}\end{array}$ & $\begin{array}{c}a \delta i_{F} \\
{[\mathrm{~m}]}\end{array}$ & $\begin{array}{c}\text { Comm. } \delta v \\
{[\mathrm{~m} / \mathrm{s}]}\end{array}$ \\
\hline far-mid & 19 -Nov-2016 15:30 & $\approx 3.5$ & $\approx 10000$ & 1000 & 100 & 60 & 0.358 \\
\hline mid-close & 24 -Nov-2016 20:00 & $\approx 2.5$ & $\approx 2500$ & $<100$ & 60 & 30 & 0.162
\end{tabular}

Having embedded all the difficulties of its task into a simple though robust algorithm, the SW implementation of OSM becomes trivial. Referring to Fig. 4, the main task (executed every $30 \mathrm{~s}$ ) consists in propagating the initial relative state $\mathbf{x}\left(t_{0}\right)$ to a prediction time (i.e., usually the current time plus 24 hours), including all the maneuvers executed since then. And, at such prediction time the criterion of Eq. 10 is checked. Whereas, if a request from the AVANTI unit occurred since the last call of the OSM application, the criterion is verified for the predicted relative state at $t_{\mathrm{M}}+24$ hours, being the time of the maneuver under evaluation later than the current time. OSM initial conditions $\mathbf{x}\left(t_{0}\right)$ have to be regularly updated from ground, using the output of the ECC activities. The frequency of re-initialization depends on number and performance of meanwhile executed maneuvers, as well as on the level of criticality of the operations (generally linked to the 3D inter-satellite separation). Standard deviations of delta-v magnitude and direction errors can also be tuned via TCs, and should represent the statistical behavior of the propulsion system. The violation of the safety criterion inhibits any further autonomous maneuver command from AVANTI, until a decision is taken from ground.

\section{In-Orbit Activities}

The commissioning of the AVANTI GNC system began shortly after the inorbit release of BEESAT-4, and in parallel to the completion of the BIROS bus validation. It comprised the stepwise verification of several interfaces and functionalities, since AVANTI formation-flying objectives required the following essential capabilities of the BIROS platform: attitude determination and control, absolute orbit determination, power/thermal/communication management, and readiness of the propulsion system. As a result, taking into account all phases of increasing authority and autonomy of the AVANTI GNC system, a total amount of two months of flight experience has been collected. Here, focus is restricted on the fully autonomous phase: the demonstration of the primary goal of the AVANTI experiment [2].

Two completely autonomous rendezvous have been performed in the second half of November 2016, exploring far- to mid- and mid- to close-range domains. Table 4 summarizes duration, covered mean along-track separation $(a \delta \lambda)$, and delta-v cost characteristics. The definition of the aimed final 
state is completed by the magnitudes of relative eccentricity ( $a \delta e)$ and of relative inclination $(a \delta i)$ vectors; a passively safe configuration is implied. With autonomous operations it is meant that only few high-level commands have been sent to BIROS, namely: target ROE-state and target time, planner operative mode (see Table 1), list of scheduled high-rate down-links, and temperature thresholds for triggering the transitions to CDM (see Table 3). In addition, as part of the safety concept, the OSM module has been periodically re-initialized, based on the ground-based pictures reprocessing [32].

\subsection{Flight Results: Far to Mid Range Rendezvous}

The first results regard autonomy, that is how the GNC system described in section 3 responded to the high-level commands reported in Table 4 . Such results are summarized in the telemetry plots of Fig. 8, where focus is given on the coordination between orbit corrections (left views) and attitude management (right views). More in details, the aimed relative state has been achieved by performing several maneuvers (see Command man state) allocated in the slots of timeline free from no-control-window constraints (i.e., gray areas in the maneuver command logic plot). These maneuvers required BIROS to be in TFM mode; whereas high-rate down-link contacts occurred approximately twice per day (see the operations concept in section 2.3) with BIROS in EPM mode. The last sub-plots on the right present the results of the thermal issues mitigation strategy: the autonomous balance between COM and CDM modes to keep BIROS temperatures inside the prescribed threshold. During the approach the CHU1 head has been used to take images; it has been switched to track the local LOS on the evening of the $21^{\text {st }}$ of November. The rotation about the boresight (i.e., second direction defining the COM profile) obeyed to the second option of Table 2, thus realizing a Sun-optimal profile (more details in Ref. 44).

Fig. 9(a) completes the overview of the behavior of the autonomous activities, since it shows how the G\&C task interacted with the onboard safety monitoring function. According to it, during the far- to mid-range rendezvous, OSM has been re-initialized approximately once per day. In addition, all the orbit corrections commanded by AVANTI have fulfilled the safety criterion of Eq. (10), with the score plotted in the bottom view. The resulting rendezvous trajectory is shown in Fig. 9(b), as seen in the RTN frame centered on the BEESAT-4 satellite. It presents a spiraling pattern around the flight-direction whose size smoothly shrinks while decreasing the inter-satellite distance. Such trajectory is the result of the employed safety concept which forces to avoid large translations in radial direction (generated by large tangential burns), which might bring the minimum RN distance value close to zero, despite magnitude and phasing of the relative eccentricity and inclination vectors.

Fig. 10 shows how ROEs evolved over time during the approach. Such 

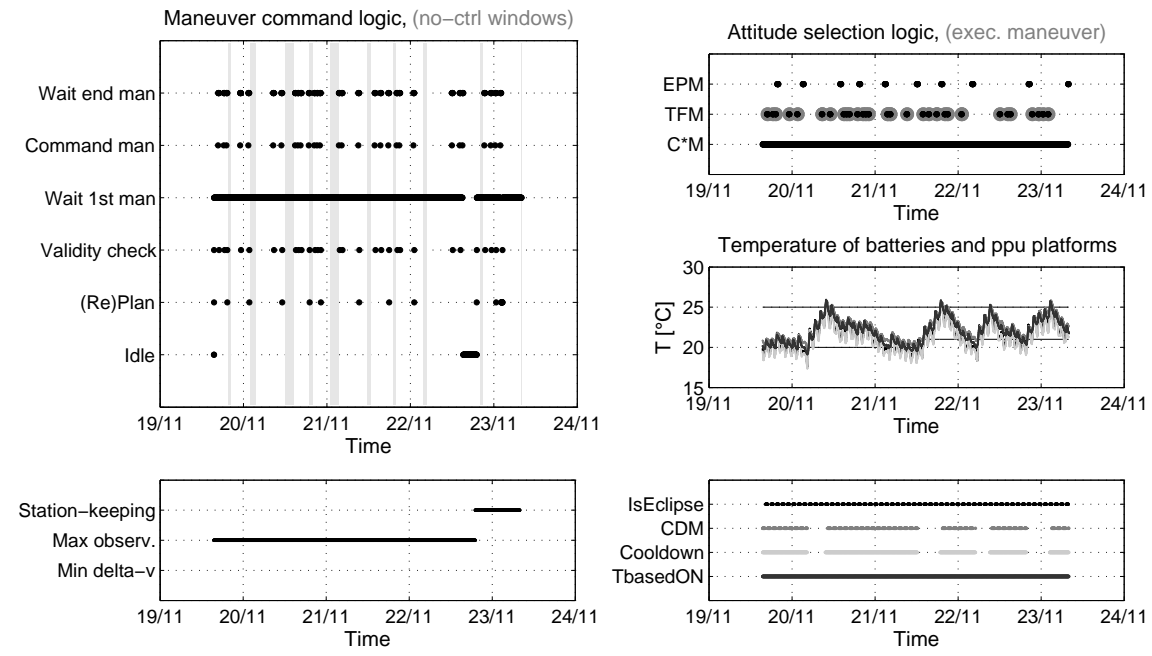

Figure 8: Autonomous coordination of orbit corrections and attitude modes.

plot encloses two levels of information: the trajectory design produced by the guidance algorithm (this time as seen in the ROE space) and the overall GNC performance. Regarding the first topic, the sequence of way-points (marked in gray) show that the relative eccentricity and inclination phasing is kept during the approach. Note that among all possible passively-safecompliant $\delta \mathbf{e} / \delta \mathbf{i}$ configurations, one with $a \delta i_{x} \approx 0$ is chosen to minimize the secular effect of the $J_{2}$ perturbation on the out-of-plane motion. Regarding the GNC accuracy, instead, the navigation error is shown as the difference between the onboard estimated ROEs (marked by black-full-circles) and the output of the ground-based relative precise orbit determination (rPOD) verification layer [32], marked by black-empty-circles. Such navigation error is sampled at the time of each plan-update, coherently with the GNC implementation scheme sketched in Fig. 4. Accordingly, the overall closed-loop GNC accuracy is given by the distance between the rPOD output and the closest targeted way-point. At $1 \mathrm{~km}$, that is at conclusion of this rendezvous, the overall GNC error stays within $\{5,1,10,200\} \mathrm{m}$ in $a\left\{\delta a, \delta *_{x}, \delta *_{y}, \delta \lambda\right\}$ components.

The navigation error contribution is predominant at the beginning of the rendezvous and at far-range. The time needed to reduce such navigation error is due to the challenging combination of few available observations, moreover confined in a limited portion of the relative orbit (unfavorable observation geometry), with the need to estimate also the effect of a varying differential aerodynamic drag (i.e., $a \delta \dot{a}$ component augmenting the state of the filter). Measurements' sparsity can be appreciated in Fig. 11(a), where the onboard observation residuals are plotted over some hours of the ren- 


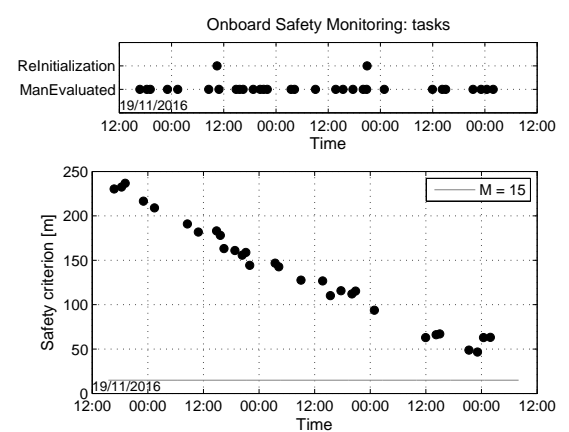

(a) Onboard safety monitoring output.

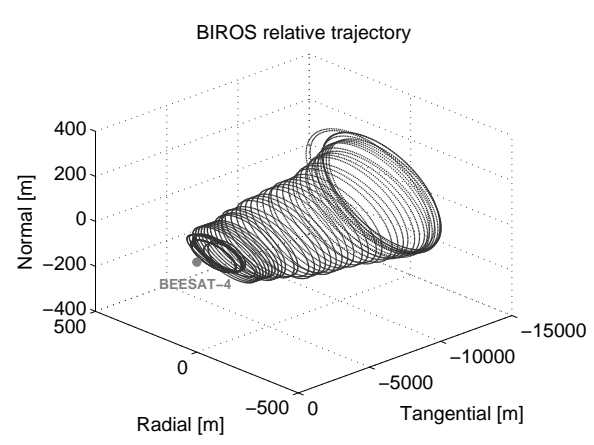

(b) Trajectory in the RTN frame centered on BEESAT-4.

Figure 9: Safety concept implementation and consequent relative trajectory.

dezvous. At far-mid range, data arcs never last more than 10 minutes, depending on the thermal phase (recall the trade-off at the end of section 3.3). Gray areas denote that the stars in background of the images could be used to refine the attitude determination of the sensor direction, thus removing measurement biases. Fig. 11(b), instead, plots BIROS impact area against the time, to provide an understanding of the varying differential drag effect. One can note that, the attitude profile induced by the CDM mode (in gray) introduces phases where BIROS is braking more (see CDM-cooldown phases in Fig. 8). In the COM profile (in black) it is clearly recognizable the transition into tracking mode (at the end of 21 Nov.); afterwards oscillations increase in amplitude to keep the target in the camera field of view while reducing the inter-satellite distance. More details regarding the target detection results and relative navigation challenges at far-mid range are discussed in Ref. 37.

From the experiment point of view, although this early navigation error in along-track degraded the lateral accuracy (see in Fig. 10 the errors in the $\mathrm{y}$-components of $\delta e$ and $\delta i$ ), the obtained trajectory remains well inside the applicability boundaries of the adopted passively safe approach, for all the time required to improve the navigation solution.

Given the adopted implementation scheme, the closed-loop control accuracy degrades due to the navigation error at re-plan, guidance assumption of keeping $a \delta \dot{a}$ constant until the next re-plan (see Eq. (14) and results in Fig. 12(a)p, and the cumulative effect of maneuver execution errors. This latter topic is addressed in Fig. 12(b), where the errors (magnitude and direction) between commanded and calibrated delta-vs are plotted against the time. Empty-circles mark very small maneuvers (i.e., $<5 \mathrm{~mm} / \mathrm{s}$ ), since these are at the edge of the GPS-based calibration capability: errors appear pretty large, though such values might not be reliable. On the other hand, cases like the last burn on the $20^{\text {th }}$ of November are critical (i.e., out-of- 

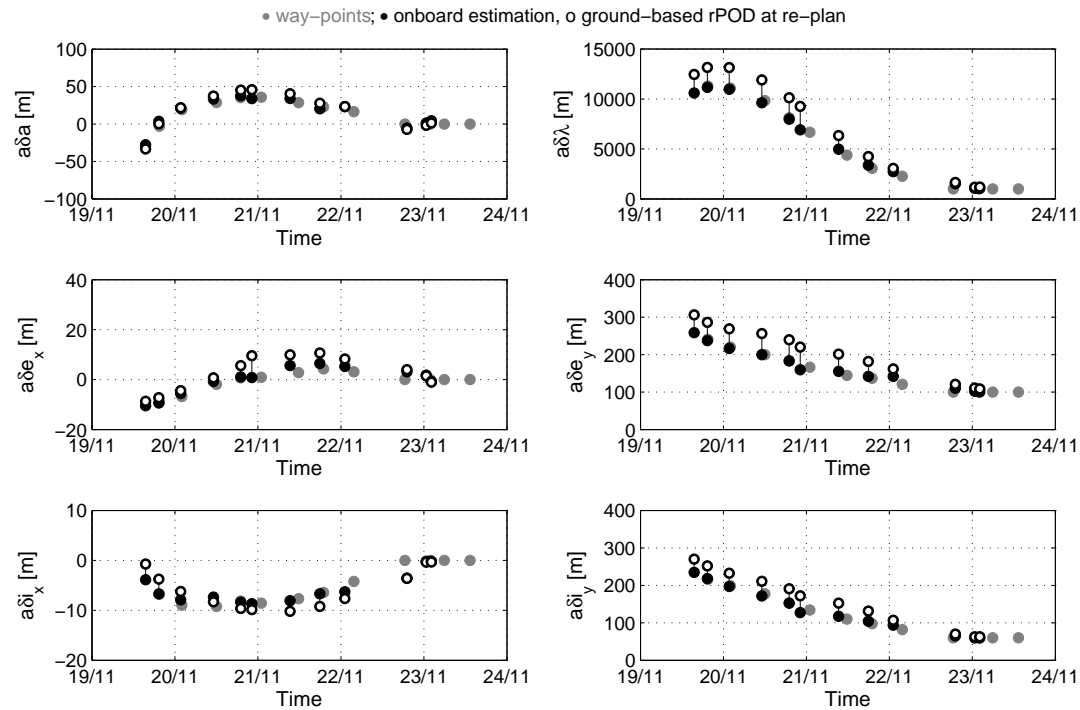

Figure 10: Relative orbit elements over time: rendezvous profile and overall GNC error.

plane maneuver of $\approx 2 \mathrm{~cm} / \mathrm{s}$ ), since they introduce a large uncertainty in the navigation filter. Recall that the onboard navigation system can only use the information of commanded maneuvers instead of the calibrated ones, and that maneuvers are used to improve the observability property of the relative navigation problem. On BIROS maneuver execution errors were mainly driven by a downgraded attitude control performance resulting from the blinding of one to two heads generated by AVANTI use of the sensor and related attitude profile (recall Fig. 6 and see Ref. 44).

\subsection{Flight Results: Mid to Close Range Rendezvous}

During the 36 hours break between the two rendezvous, the action of the differential drag perturbation brought BIROS back again to a separation of circa $2.5 \mathrm{~km}$. The objective of this second approach was to reach the minimum 3D distance achievable given operations safety limits and system's restrictions (clearly listed in section 3 of Ref. 45). This goal explains the definition of the final ROE-state in Table 4; the target relative inclination magnitude has been reduced to $30 \mathrm{~m}$ and $\delta \lambda_{F}$ and $\delta e_{F}$ have been chosen to allow BIROS overtaking BEESAT-4 during the portion of relative orbit spent in eclipse.

The so obtained rendezvous profile is shown in Fig. 13 and the overall GNC error stays within $\{0.5,1,1,10\} \mathrm{m}$ in $a\left\{\delta a, \delta *_{x}, \delta *_{y}, \delta \lambda\right\}$ components at the conclusion of the approach. In this phase no-control-window con- 


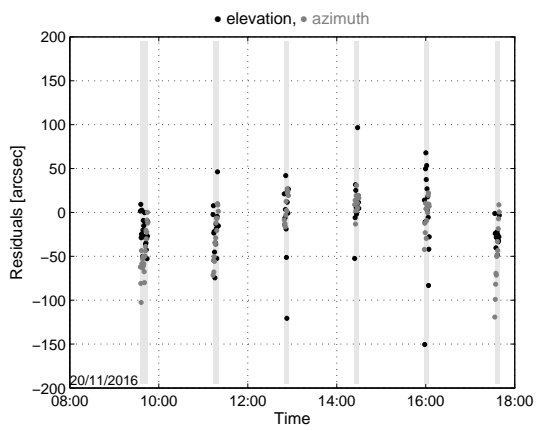

(a) Onboard observation residuals.

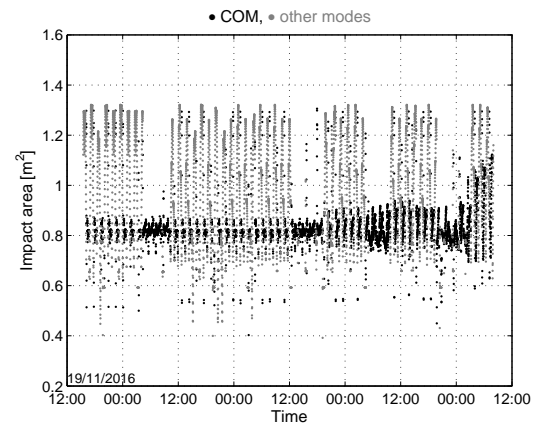

(b) Variation of the impact area.

Figure 11: Contributions to the guidance and control error.

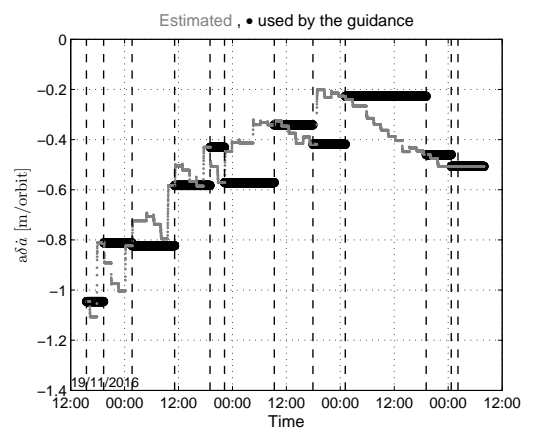

(a) Onboard handling of $a \delta \dot{a}$.

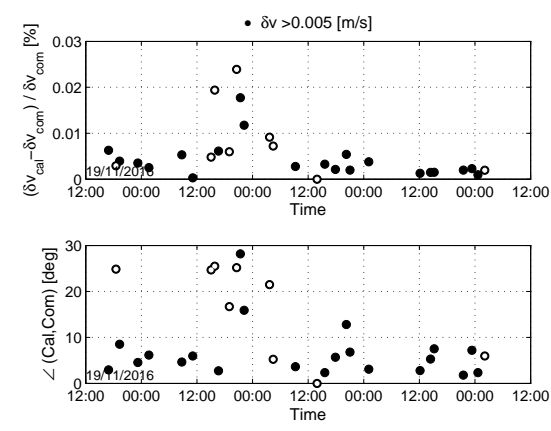

(b) Maneuver execution errors.

Figure 12: Contributions to the guidance and control error.

straints and minimum delta-v operative mode are used to get more sparse way-points: the natural drift is exploited considering that the implemented relative orbit control cannot structurally achieve a fine continuous tracking and maneuvers determine picture data-gaps. In addition, the aimed final time of the mid-close range rendezvous has been chosen to synchronize natural drifting phases with a period in which no heat dissipation interruptions are required.

As a result, several close-range pictures of BEESAT-4 could be retrieved (examples in Fig. 14(a)p; depending on the luminosity conditions, some of them show coarse shape details (e.g., geometry and antenna tips) others are more blurred. The complete rendezvous trajectory, as seen in the RTN frame centered on BEESAT-4, is presented in Fig. 14(b), where the location of the observations are marked in black. Note that in the close vicinity of the target, thanks to the prevalence of the out-of-plane component, definitely more observations (up to 30 minutes) could be imaged, especially when no conflicts with maneuvers, data-links, and CDM occurred. 

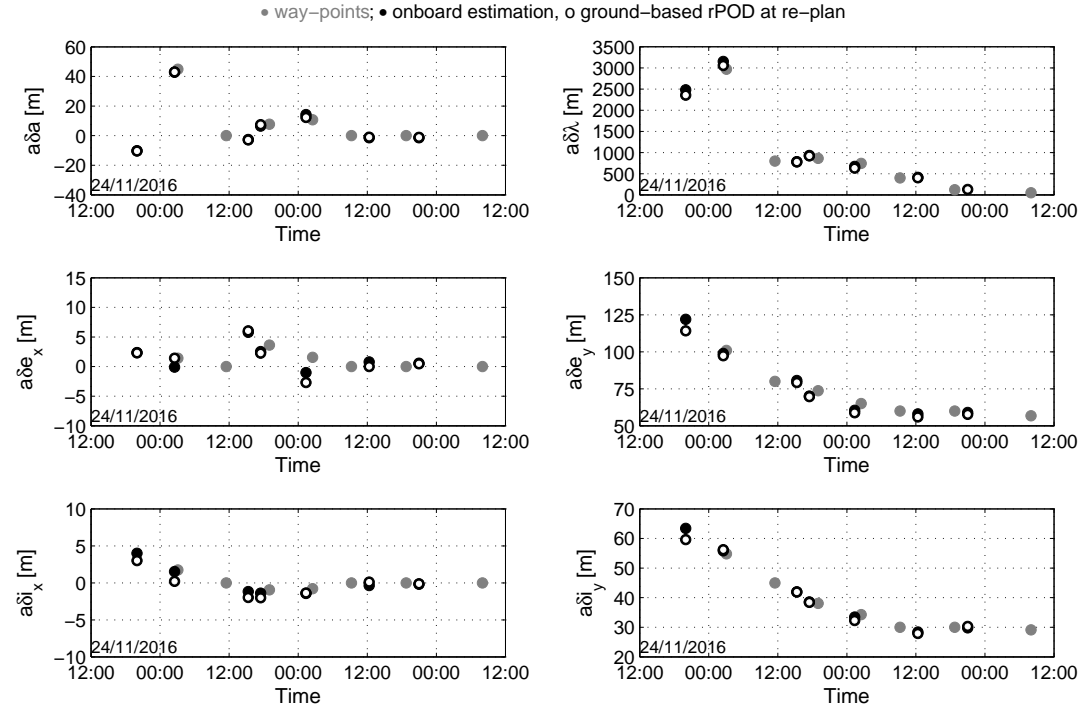

Figure 13: Relative orbit elements over time: rendezvous profile and overall GNC error.
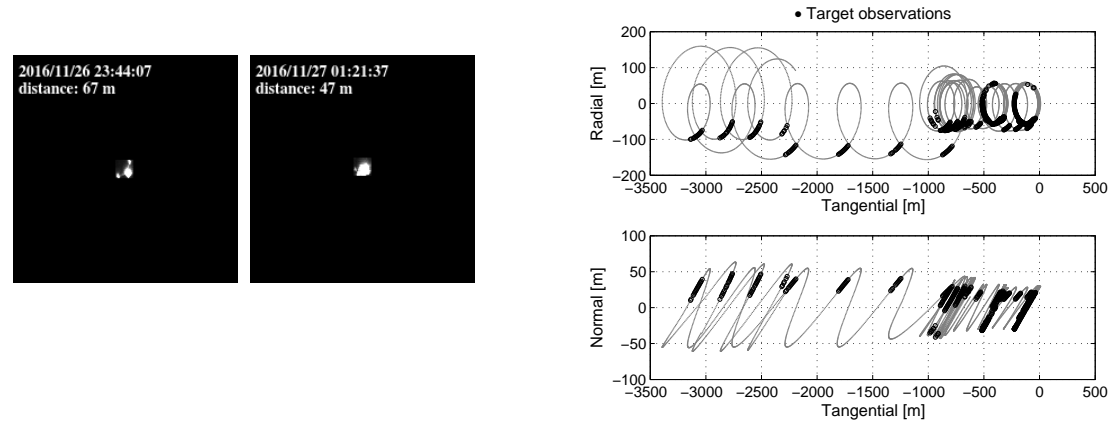

(a) ROIs containing the target with and without electronic shut-

(b) RT - NT views of the trajectory. ter.

Figure 14: Close-range approach. 
The close-range region constituted the most challenging condition for the GNC system of AVANTI, which relies only on a far-range camera and has already to cope with several operational constraints (e.g., single-direction thruster system, 30 seconds of fastest image-rate, conflicting pointing requirements). Regarding the collection of images, with the decrease of the inter-satellite distance it became mandatory to use the electronic shutter to limit the exposure time, and thus to reduce the centroiding error (i.e., distance between center of mass and luminous center, also related to image cuttings generated by the fixed-area ROI compression). As side-effect, the images did not show anymore stars in background, since their brightness was too weak compared to the one of the target. Basically, the centroiding error is reduced at the cost of losing an accurate information of the pointing direction of the camera sensor. Fig. 15 shows such effects on the observation residuals: black values are achieved when stars are available in background to refine the sensor attitude determination; gray values are obtained using the BIROS onboard attitude estimate. The light-gray areas denote phases with active shutter. One can note that, from the one hand, the attitude refinement becomes rarer and, below $\approx 300 \mathrm{~m}$, possible only when the shutter was off. From the other hand, the BIROS attitude determination was accurate only to the degree level, thus producing residuals an order of magnitude larger. At the practical level, such phenomena had firstly been investigated during the close-range commissioning phase of AVANTI [44, and the fully autonomous rendezvous benefited from such experience employing a special tuning of the parameters of the navigation filter as discussed in Ref. 37 .

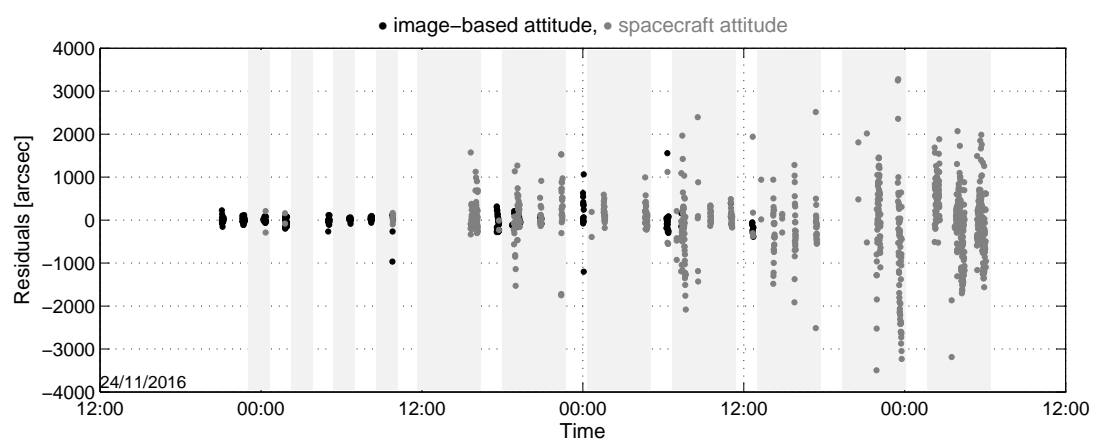

Figure 15: Onboard observation residuals at mid- to close-range.

In addition to the aforementioned optical effects, differential aerodynamic drag and maneuvers execution errors also contributed to challenge the onboard navigation system, making it difficult to point into the target direction with the accuracy required to keep BEESAT-4 in the field of view of the far-range camera. As pointed out in Ref. 44, the limited available pictures data-rate, together with the fact that the COM attitude profile is generated propagating over the AVANTI time-step the current onboard nav- 
igation solution, reduced the level of robustness achievable at close-range.

Nevertheless, despite all these difficulties, the peculiar architecture of the AVANTI GNC system customized to deal with angles-only observations, allowed reaching safely a region below $50 \mathrm{~m}$ of distance with respect to the fully uncooperative BEESAT-4 target.

\section{Conclusion}

This paper presented system design, implementation, and flight results of the spaceborne guidance, navigation, and control system developed to perform the AVANTI experiment. This endeavor demonstrated the first autonomous rendezvous to a fully noncooperative object in low Earth orbit relying exclusively on angles-only observations extracted from pictures collected by a monocular, far-range, camera system. Within AVANTI, the BIROS spacecraft successfully approached down to less than 50 meters of inter-satellite distance the passive one-unit CubeSat BEESAT-4, previously deployed in space through a single picosatellite launcher.

AVANTI pursued a low-cost, minimalistic, design approach with no impact on the configuration of the chasing spacecraft: BIROS already featured a propulsion system and a star-tracker sensor. This latter played the role of the primary relative navigation sensing instrument, being re-used as farrange camera. Although AVANTI benefited from know-how and flight experience from the precursor, ground-in-the-loop, ARGON demonstration, both experiment design approach and mission scenario posed several constraints to the spaceborne GNC system. These remarkably impacted its design in terms of architecture and implemented algorithms. The major challenges to be overcome concerned observations' sparseness and orbital perturbations, for the relative navigation system, and autonomous management of the chaser spacecraft to perform a safe rendezvous, for the guidance and control system. The resulting framework can be seen as a worst-case condition against which proving the feasibility of the angles-only approach. Since autonomy, software flexibility, applicability to various orbit environments, and inclusion of a robust safety concept are indeed fundamental requirements for future robotic rendezvous missions in low-Earth orbits.

At the same time, in view of future applications of the AVANTI approach, margins of improvement in terms of GNC system robustness a/o overall achievable accuracy are possible by removing some of the aforementioned platform constraints. First, it would be convenient to allow a larger onboard data-budget, to increase the frequency of image data a/o to relax the usage of the region-of-interest image compression format. A second structural improvement could be achieved by employing a more advanced onboard processor to support larger computational capabilities. In this way, in fact, one could consider different, or complementary, filtering techniques, 
also based on numerical integration. The absolute navigation system could be set to estimate onboard the executed maneuvers, to further enhance the observability properties of the relative navigation problem. Finally, more accurate guidance and control strategies would become feasible. Specifically for the close-range domain, a robuster system architecture would be realized by linking the target-pointing attitude guidance to the target detection task instead of to the onboard relative navigation solution. In this way, in fact, a downgraded relative navigation accuracy would not compromise the possibility to obtain further observations, despite the narrow field-of-view of the camera. Finally, in the close-range vicinity of the target the 3D maneuver capability is an asset to safely reduce the inter-satellite distance.

The absence of any form of cooperation between chaser and target represents the major peculiarity of the AVANTI demonstration. This not only impacted onboard safety management and rendezvous guidance policy, but also the post-facto assessment of the true experiment performances. In this frame, further investigation is needed to quantify the in-orbit differential drag effects and to evaluate more accurately the capability of the navigation filter to catch them. The major difficulties in carrying out such activities are given by the need of isolating different navigation disturbance and error contributions.

Despite all the aforementioned limitations, AVANTI embodies a milestone in the preparation of future debris removal missions, since it demonstrated the viability of the autonomous angles-only relative navigation approach into an extremely challenging, and representative, orbit scenario. Developed technology and collected flight data will support the preparation of future DLR activities on the roadmap to develop and realize on-orbitservicing and active debris removal.

\section{Appendix}

\subsection{Relative orbit guidance problem}

This appendix presents the explicit form solution of the relative orbit guidance problem. The evolution of the relative state to achieve $\mathbf{x}_{F}$ through $m$ intermediate steps expressed in Eq. (5) can be reordered according to:

$$
\left[\begin{array}{llll}
\boldsymbol{\Phi}_{F, 1} & \cdots & \boldsymbol{\Phi}_{F, m-1} & \boldsymbol{\Phi}_{F, m}
\end{array}\right]\left(\begin{array}{c}
\Delta x_{1} \\
\Delta x_{2} \\
\vdots \\
\Delta x_{m}
\end{array}\right)=\mathbf{b}_{0}=\left(\mathbf{x}_{F}-\boldsymbol{\Phi}_{F, 0} \mathbf{x}_{0}\right)
$$

where the compact notation $\boldsymbol{\Phi}_{F, i}=\boldsymbol{\Phi}\left(t_{F}, t_{i}\right)$ is used. Here, the unknown ROE changes determined by the effect of $m$-groups of impulsive maneuvers are multiplied by known quantities, once having fixed the sequence of times 
$\left(t_{1}, \cdots, t_{m}, t_{F}\right)$. The right member $\mathbf{b}_{0}$ collects the whole effect of the natural perturbed dynamics over the total guidance horizon time $\left(t_{F}-t_{0}\right)$. At this point, it is convenient to further re-arrange Eq. (11) grouping the $m$ variations of each $\mathrm{ROE}$ element (from the $p-t h$ to the $q-t h$ element):

$$
\begin{aligned}
& {\left[\begin{array}{llllll|llll}
\boldsymbol{\Phi}_{F, 1}^{\mathrm{p}-\mathrm{col}} & \ldots & \boldsymbol{\Phi}_{F, m-1}^{\mathrm{p}-\mathrm{col}} & \boldsymbol{\Phi}_{F, m}^{\mathrm{p}-\mathrm{col}} & \cdots & \boldsymbol{\Phi}_{\mathrm{F}, 1}^{\mathrm{q}-\mathrm{col}} & \cdots & \boldsymbol{\Phi}_{\mathrm{F}, m-1}^{\mathrm{q}-\mathrm{col}} & \mathbf{\Phi}_{\mathrm{F}, m}^{\mathrm{q}-\mathrm{col}}
\end{array}\right]\left(\begin{array}{c}
\Delta x_{p, 1} \\
\vdots \\
\Delta x_{p, m} \\
\vdots \\
\hline \Delta x_{q, 1} \\
\vdots \\
\Delta x_{q, m}
\end{array}\right)} \\
& =\left(\begin{array}{c}
b_{0, p} \\
\vdots \\
b_{0, q}
\end{array}\right)
\end{aligned}
$$

The satisfaction of the end-conditions at the final time (i.e., achievement of $\mathbf{x}_{F}$ ) can be simply enforced by writing the last ROE variation $\Delta \mathbf{x}_{m}$ as function of the previous $m-1$ corrections applied to the initial state $\mathbf{x}_{0}$. Subsequently, the optimal (i.e., delta-v minimum) $m-1$ ROE corrections are computed by enforcing the following necessary conditions for optimality of the convex problem with cost function Eq. (8):

$$
\frac{\partial J_{\text {plan }}}{\partial \Delta \tilde{\mathbf{x}}_{j}}=\mathbf{0}_{1 \times m-1}^{\mathrm{T}} \quad j=p, \cdots, q
$$

which determine $q-p$ equations for the $m-1$ corrections of each element (i.e., $\left.\Delta \tilde{\mathbf{x}}_{j}=\left(\Delta x_{j, 1} \cdots \Delta x_{j, m-1}\right)^{\mathrm{T}}\right)$. By exploiting the block-structure of the state transition matrix of Eq. (6), Eq. (13) reduces to two decoupled sub-problems respectively in the $\left(\delta \dot{a}, \delta a, \delta \lambda, \delta i_{x}, \delta i_{y}\right)$ and $\left(\delta e_{x}, \delta e_{y}\right)$ spaces.

For the along/cross-track planning sub-problem, it is assumed that maneuvers do not determine changes in the mean relative semi-major axis timevariation due to differential aerodynamic drag (i.e., $\Delta \mathbf{x}_{1}=0$ ). Therefore the optimal corrections of the $\left(\delta a, \delta \lambda, \delta i_{x}, \delta i_{y}\right)$ elements, solution of Eq. (13) 
with $p=2$ and $q=5$, are given by the following linear system:

$$
\begin{aligned}
\left(\begin{array}{c}
\Delta \tilde{\mathbf{x}}_{2} \\
\vdots \\
\Delta \tilde{\mathbf{x}}_{5}
\end{array}\right)_{o p t}= & {\left[\begin{array}{cccc}
\mathbf{I}+\mathbf{A}+\left(\mu_{N}^{2}+\lambda_{A}^{2}\right) \mathbf{D} & \mu_{N} \mathbf{C} & \left(\mu_{N} \mu_{I}+\lambda_{A} \lambda_{I}\right) \mathbf{D} & \lambda_{A} \mathbf{C} \\
\mu_{N} \mathbf{C}^{\mathrm{T}} & \mathbf{I}+\mathbf{A} & \mu_{I} \mathbf{C}^{\mathrm{T}} & \mathbf{O} \\
\left(\mu_{N} \mu_{I}+\lambda_{A} \lambda_{I}\right) \mathbf{D} & \mu_{I} \mathbf{C} & \mathbf{I}+\mathbf{A}+\left(\mu_{I}^{2}+\lambda_{I}^{2}\right) \mathbf{D} & \lambda_{I} \mathbf{C} \\
\lambda_{A} \mathbf{C}^{\mathrm{T}} & \mathbf{O} & \lambda_{I} \mathbf{C}^{\mathrm{T}} & \mathbf{I}+\mathbf{A}
\end{array}\right]^{-1} } \\
& \left(\begin{array}{c}
b_{0,2} \tilde{\mathbf{a}}+\left(\mu_{N} b_{0,3}^{*}+\lambda_{A} b_{0,5}^{*}\right) \tilde{\mathbf{d}} \\
b_{0,3}^{*} \tilde{\mathbf{a}} \\
b_{0,4} \tilde{\mathbf{a}}+\left(\mu_{I} b_{0,3}^{*}+\lambda_{I} b_{0,5}^{*}\right) \tilde{\mathbf{d}} \\
b_{0,5}^{*} \tilde{\mathbf{a}}
\end{array}\right) \\
b_{0,3}^{*}= & b_{0,3}-\mu_{N} b_{m} b_{0,2}-\mu_{I} b_{m} b_{0,4} \\
b_{0,5}^{*}= & b_{0,5}-\lambda_{A} b_{m} b_{0,2}-\lambda_{I} b_{m} b_{0,4}
\end{aligned}
$$

where $\mu_{N}=\nu+\mu_{A}$, and the matrix quantities are $\mathbf{A}=\tilde{\mathbf{a}} \tilde{\mathbf{a}}^{\mathrm{T}}, \mathbf{D}=\tilde{\mathbf{d}} \tilde{\mathbf{d}}^{\mathrm{T}}$, $\mathbf{C}=\tilde{\mathbf{d}} \tilde{\mathbf{a}}^{\mathrm{T}}$, obtained from these service vector quantities

$$
\tilde{\mathbf{a}}=\mathbf{I}_{m-1 \times 1} \quad \mathbf{b}_{m \times 1}=\left(\begin{array}{c}
t_{F}-t_{1} \\
\vdots \\
t_{F}-t_{m}
\end{array}\right)=\left(\begin{array}{c}
\tilde{\mathbf{b}} \\
b_{m}
\end{array}\right) \quad \tilde{\mathbf{d}}=\tilde{\mathbf{b}}-b_{m} \tilde{\mathbf{a}}
$$

where $\tilde{\bullet}$ labels vectors of dimension $m-1$.

For the relative eccentricity vector planning sub-problem, the optimal corrections of the $\delta e_{x}$ and $\delta e_{y}$ components, solution of Eq. (13) with $p=6$ and $q=7$, are given by the following linear system:

$$
\begin{aligned}
\left(\begin{array}{c}
\Delta \tilde{\mathbf{x}}_{6} \\
\Delta \tilde{\mathbf{x}}_{7}
\end{array}\right)_{o p t} & =\left[\begin{array}{cc}
\mathbf{I}+\mathbf{F}+\mathbf{G} & \mathbf{H}-\mathbf{H}^{\mathrm{T}} \\
\mathbf{H}^{\mathrm{T}}-\mathbf{H} & \mathbf{I}+\mathbf{F}+\mathbf{G}
\end{array}\right]^{-1}\left(\begin{array}{c}
-b_{0,6}^{*} \tilde{\mathbf{f}}+b_{0,7}^{*} \tilde{\mathbf{g}} \\
-b_{0,6}^{*} \tilde{\mathbf{g}}-b_{0,7}^{*} \tilde{\mathbf{f}}
\end{array}\right) \\
b_{0,6}^{*} & =+c_{m} b_{0,6}+s_{m} b_{0,7} \\
b_{0,7}^{*} & =-s_{m} b_{0,6}+c_{m} b_{0,7}
\end{aligned}
$$

where the matrix quantities are $\mathbf{F}=\tilde{\mathbf{f}} \tilde{\mathbf{f}}^{\mathrm{T}}, \mathbf{G}=\tilde{\mathbf{g}} \tilde{\mathbf{g}}^{\mathrm{T}}, \mathbf{H}=\tilde{\mathbf{f}} \tilde{\mathbf{g}}^{\mathrm{T}}$, having defined the following vector quantities:

$$
\begin{aligned}
& \mathbf{c}_{m \times 1}=\left(\begin{array}{c}
\cos \left(\dot{\varphi}\left(t_{F}-t_{1}\right)\right) \\
\vdots \\
\cos \left(\dot{\varphi}\left(t_{F}-t_{m}\right)\right)
\end{array}\right)=\left(\begin{array}{c}
\tilde{\mathbf{c}} \\
c_{m}
\end{array}\right) \\
& \mathbf{s}_{m \times 1}=\left(\begin{array}{c}
\sin \left(\dot{\varphi}\left(t_{F}-t_{1}\right)\right) \\
\vdots \\
\sin \left(\dot{\varphi}\left(t_{F}-t_{m}\right)\right)
\end{array}\right)=\left(\begin{array}{c}
\tilde{\mathbf{s}} \\
s_{m}
\end{array}\right)
\end{aligned}
$$




\subsection{Maneuvers computation}

This appendix presents the closed-form computation of the impulsive deltavs to establish the $\mathbf{x}_{i+1}$ way-point starting from its $\mathbf{x}_{i}$ precursor. This $i-t h$ local problem can be written as:

$$
\left[\begin{array}{lll}
\boldsymbol{\Phi}_{i+1,1}^{\mathrm{HCW}} \mathbf{B}_{1} & \cdots & \boldsymbol{\Phi}_{i+1, w}^{\mathrm{HCW}} \mathbf{B}_{w}
\end{array}\right]\left[\begin{array}{c}
\delta \mathbf{v}_{1} \\
\vdots \\
\delta \mathbf{v}_{w}
\end{array}\right]=\left(\mathbf{x}_{i+1}-\boldsymbol{\Phi}_{i+1, i} \mathbf{x}_{i}\right)=\mathbf{b}_{i}
$$

where $\mathbf{B}$ is the control input matrix which expresses the effects of an impulsive maneuver in the orbital RTN frame performed at time $t_{j}$, and thus at mean argument of latitude $u_{j}$, on the state vector of Eq. (3):

$$
\mathbf{B}\left(t_{j}\right)=\mathbf{B}_{j}=-\frac{1}{n}\left[\begin{array}{ccc}
0 & 0 & 0 \\
0 & 2 & 0 \\
-2 & 0 & 0 \\
0 & 0 & \cos u_{j} \\
0 & 0 & \sin u_{j} \\
\sin u_{j} & 2 \cos u_{j} & 0 \\
-\cos u_{j} & 2 \sin u_{j} & 0
\end{array}\right]
$$

In Eq. (18), $w$ delta-vs are used to achieve the effective ROE change $\mathbf{b}_{i}$, to the net of the perturbations acting over the $t_{i+1}-t_{i}$ reconfiguration time horizon. In order to obtain an analytical solution, the approximation of using the Hill-Clohessy-Wiltshire state transition matrix to pre-multiply $\mathbf{B}$ is introduced, thus neglecting the perturbations differential effect on the changes in the relative orbits operated by the intermediate maneuvers. As a matter of fact, these contributions determine negligible deviations in the mean arguments of latitude of the computed maneuvers, for reconfigurations over few orbits time. The resulting structure of the first matrix of Eq. (18), on the contrary, discloses that the in-plane and out-of-plane motions are decoupled. The latter requires a single maneuver in normal direction placed either at a $u_{\mathrm{N}}$ or $u_{\mathrm{N}}+\pi$ opportunity according to:

$$
u_{\mathrm{N}}=\arctan \left(\frac{b_{i, 5}}{b_{i, 4}}\right) \quad \text { with } \quad \delta v_{\mathrm{N}}=n \sqrt{b_{i, 4}^{2}+b_{i, 5}^{2}}
$$

where the sign of the $\delta v_{\mathrm{N}}$ is chosen to satisfy the $\left(b_{i, 4}, b_{i, 5}\right)^{\mathrm{T}}$ end-conditions. The in-plane problem, once fixed to use three tangential pulses, presents at least one analytical solution, upon the condition of placing the maneuvers at half orbital period multiples of the mean argument of latitude $\bar{u}$ that equals the phase angle of the whole variation of the relative eccentricity vector:

$$
\bar{u}=\arctan \left(\frac{b_{i, 7}}{b_{i, 6}}\right), u_{\mathrm{T} j}=\bar{u}+k_{j} \pi, j=1 \ldots 3, k_{1}<k_{2}<k_{3}
$$


Chosen a $k_{j}$ sequence that fits the $t_{i+1}-t_{i}$ time elapse, the corresponding delta-vs are obtained enforcing the remaining end-conditions as:

$$
\left[\begin{array}{ccc}
2 & 2 & 2 \\
-3\left(u_{i}-u_{\mathrm{T} 1}\right) & -3\left(u_{i}-u_{\mathrm{T} 2}\right) & -3\left(u_{i}-u_{\mathrm{T} 3}\right) \\
2 \cos u_{\mathrm{T} 1} & 2 \cos u_{\mathrm{T} 2} & 2 \cos u_{\mathrm{T} 3}
\end{array}\right]\left(\begin{array}{c}
\delta v_{\mathrm{T} 1} \\
\delta v_{\mathrm{T} 2} \\
\delta v_{\mathrm{T} 3}
\end{array}\right)=-n\left(\begin{array}{c}
b_{i, 2} \\
b_{i, 3} \\
b_{i, 6}
\end{array}\right)
$$

if $\cos \bar{u} \neq 0$, otherwise the equation in $b_{i, 7}$ has to replace the one in $b_{i, 6}$.

\section{Acknowledgments}

The construction of the BIROS satellite was funded by the Federal Ministry of Education and Research of Germany (BMBF), project number FKZ 01LK0904A.

The Authors would like to thank the DLR Optical Sensor Systems Team, for their support during SW integration and validation on BIROS and during the experiment commissioning in orbit; the GSOC Mission Operations Team, for their assistance during flight operations; the GSOC Flight Dynamics Services Team, for their support after BEESAT-4 deployment and during the conduction of the AVANTI experiment.

\section{References}

[1] "The AVANTI weblog," http://www.dlr.de/rb/desktopdefault. aspx/tabid-11685/\#gallery/28310, DLR Raumflugbetrieb und Astronautentraining, Dec. 2016, [retrieved 4 August 2017].

[2] Gaias, G., Ardaens, J.-S., and D'Amico, S., "The Autonomous Vision Approach Navigation and Target Identification (AVANTI) Experiment: Objectives and Design," Proceedings of the $9^{\text {th }}$ International ESA Conference on Guidance, Navigation $\&$ Control Systems, European Space Agency, ESTEC, Noordwijk, The Netherlands, 2014.

[3] Weismuller, T. and Leinz, M., "GN\&C Technology Demonstrated by the Orbital Express Autonomous Rendezvous and Capture Sensor System," No. Paper 06-016, 29 ${ }^{\text {th }}$ Annual AAS Guidance and Control Conference, American Astronautical Society, 2006.

[4] "DARPA archive: Orbital Express On-Orbit Mission Updates," http: //archive.darpa.mil/orbitalexpress/mission_updates.html, DARPA Tactical Technology Office, July 2007, [retrieved 4 August 2017].

[5] Jørgensen, J. L., Denver, T., and Jørgensen, P. S., "Using an Autonomous Star Tracker as Formation Flying Sensor," Proceedings of 
the 4 S Symposium: Small Satellites, Systems and Services, 20 - 24 September 2004, La Rochelle, France, ESA Publications Division (ESA SP-571, October 2004), ESTEC, Noordwijk, The Netherlands, 2004.

[6] Noteborn, R., Bodin, P., Larsson, R., and Chasset, C., "Flight Results from the PRISMA Optical Line of Sight Based Autonomous Rendezvous Experiment," Proceedings of the $4^{\text {th }}$ International Conference on Spacecraft Formation Flying Missions \& Technologies (SFFMT), Canadian Space Agency, St-Hubert, Quebec, 2011.

[7] Karlsson, T., Ahlgren, N., Faller, R., and Schlepp, B., "PRISMA Mission Control: Transferring Satellite Control between Organisations," SpaceOps 2012, Stockholm, Sweden, American Institute of Aeronautics and Astronautics, 2012, doi.org/10.2514/6.2012-1287645.

[8] Faller, R., Schlepp, B., Gaias, G., Ohndorf, A., and Kruse, W., "PRISMA Betrieb am GSOC - Schlussbericht," PRI-GSO-RPT-018, Deutsches Zentrum für Luft- und Raumfahrt, Oberpfaffenhofen, Germany, Feb. 2013.

[9] Delpech, M., Berges, J.-C., Djalal, S., and Christy, J., "Vision Based Rendezvous Experiment performed during the PRISMA Extended Mission," Proceedings of the 23rd International Symposium on Space Flight Dynamics, Jet Propulsion Laboratory, Pasadena, California, USA, 2012.

[10] D'Amico, S., Ardaens, J.-S., Gaias, G., Benninghoff, H., Schlepp, B., and Jørgensen, J. L., "Noncooperative Rendezvous Using Angles-Only Optical Navigation: System Design and Flight Results," Journal of Guidance, Control, and Dynamics, Vol. 36, No. 6, 2013, pp. 1576-1595, doi: $10.2514 / 1.59236$.

[11] Karlsson, T., Larsson, R., Jakobsson, B., Bodin, P., and Larsson, B., "The IRIDES Experiment: PRISMA Rendezvous with the noncooperative Satellite Picard," Proceedings of the 4 S Symposium: Small Satellites, Systems and Services, 26 - 30 May 2014, Majorca, Spain, European Space Agency, Noordwijk, The Netherlands, 2014.

[12] Karlsson, T., Larsson, R., Jakobsson, B., Bodin, P., and Larsson, B., "PRISMA IRIDES: Performance at the End of the Drift Phase \& Planned Rendezvous Experiments," Proceedings of the $9^{\text {th }}$ International ESA Conference on Guidance, Navigation \& Control Systems, European Space Agency, ESTEC, Noordwijk, The Netherlands, 2014.

[13] Karlsson, T., "IRIDES ESA Executive Summary SSPO6091," PRISMA-OSE-RP-0003, European Space Agency, Keplerlaan 1, Noordwijk, The Netherlands, Dec. 2014. 
[14] Larsson, R., Mueller, J., Thomas, S., Jakobsson, B., and Bodin, P., "Orbit Constellation Safety on the PRISMA In-Orbit Formation Flying Test Bed," Proceedings of the $3^{\text {rd }}$ International Symposium on Formation Flying, Missions and Technologies, ESA Communication Production Office (ESA SP-654), ESTEC, Noordwijk, The Netherlands, 2008 .

[15] Gaias, G. and Ardaens, J.-S., "Design challenges and safety concept for the AVANTI experiment," Acta Astronautica, Vol. 123, 2016, pp. 409419, doi: $10.1016 /$ j.actaastro.2015.12.034.

[16] Gaias, G., Ardaens, J.-S., and Montenbruck, O., "Model of J2 Perturbed Satellite Relative Motion with Time-Varying Differential Drag," Celestial Mechanics and Dynamical Astronomy, Vol. 123, No. 4, 2015, pp. 411-433, doi: 10.1007/s10569-015-9643-2.

[17] Yamanaka, K. and Ankersen, F., "New State Transition Matrix for Relative Motion on an Arbitrary Elliptical Orbit," Journal of Guidance, Control and Dynamics, Vol. 25, No. 1, 2002, pp. 60-66.

[18] D'Amico, S., Ardaens, J.-S., and Larsson, R., "Spaceborne Autonomous Formation-Flying Experiment on the PRISMA Mission," Journal of Guidance, Control, and Dynamics, Vol. 35, No. 3, 2012, pp. 834-850, doi: $10.2514 / 1.55638$.

[19] Ardaens, J.-S., Kahle, R., and Schulze, D., "In-Flight Performance Validation of the TanDEM-X Autonomous Formation Flying System," Proceedings of the $5^{\text {th }}$ International Conference on Spacecraft Formation Flying Missions \& Technologies (SFFMT), DLR, German Space Operations Center, Munich, Germany, 2013.

[20] Gaias, G., D’Amico, S., and Ardaens, J.-S., "Generalized MultiImpulsive Maneuvers for Optimum Spacecraft Rendezvous in NearCircular Orbit," Int. J. Space Science and Engineering, Vol. 3, No. 1, 2015, pp. 68-88, doi: 10.1504/IJSPACESE.2015.069361.

[21] Ardaens, J.-S. and Gaias, G., "Spaceborne Autonomous Vision-Based Navigation System for AVANTI," No. IAC-14-B4.7B.3, 65 $5^{\text {th }}$ International Astronautical Congress, Toronto, Canada, 2014.

[22] Montenegro, S. and Bärwald, W., "PowerBIRD - Modern Spacecraft Bus Controller," Acta Astronautica, Vol. 52, No. 9-12, 2003, pp. 957963.

[23] Gaias, G., Ardaens, J.-S., and Terzibaschian, T., "Paving the Way for Future On-Orbit-Servicing Missions: the AVANTI Experiment," Proceedings of the $25^{\text {th }}$ International Symposium on Space Flight Dynam- 
ics ISSFD, DLR, German Space Operations Center, Munich, Germany, 2015.

[24] Reile, H., Lorenz, E., and Terzibaschian, T., "The FireBird Mission A Scientific Mission for Earth Observation and Hot Spot Detection," Small Satellites for Earth Observation, Digest of the $9^{\text {th }}$ International Symposium of the International Academy of Astronautics, Wissenschaft und Technik Verlag, Berlin, Germany, 2013, pp. 184-196, ISBN 978-389685-574-9.

[25] "Technische Universität Berlin, BEESAT-4 website," https: //www.raumfahrttechnik.tu-berlin.de/menue/forschung/ aktuelle_projekte/beesat-4/parameter/en/, Sascha Weiss, TU Berlin, May 2017, [retrieved 4 August 2017].

[26] Wermuth, M., Gaias, G., and D'Amico, S., "Safe Picosatellite Release from a Small Satellite Carrier," Journal of Spacecraft and Rockets, Vol. 52, No. 5, 2015, pp. 1338-1347, doi: 10.2514/1.A33036.

[27] Wermuth, M. and Gaias, G., "Operational Concept of a Picosatellite Release from a LEO Satellite," Proceedings of the $25^{\text {th }}$ International Symposium on Space Flight Dynamics ISSFD, DLR, German Space Operations Center, Munich, Germany, 2015.

[28] Föckersperger, S., Lattner, K., Kaiser, C., Eckert, S., Bärwald, W., Ritzmann, S., Mühlbauer, P., Turk, M., and Willemsen, P., "The Modular German Microsatellite TET-1 for Technology On-Orbit Verification," No. IAC-08.B4.7.3, 59 ${ }^{\text {st }}$ International Astronautical Congress, Glasgow, UK, 2008.

[29] Maibaum, O., Terzibaschian, T., Raschke, C., and Gerndt, A., "Software Reuse of the BIRD ACS for the TET Satellite Bus," Small Satellites for Earth Observation, Digest of the $8^{\text {th }}$ International Symposium of the International Academy of Astronautics, Wissenschaft und Technik Verlag, Berlin, Germany, 2011, pp. 409-412, ISBN 978-89685-573-2.

[30] Montenbruck, O. and Markgraf, M., "User's Manual for the Phoenix GPS Receiver, Ver. 1.9," Gtnman-0120, Deutsches Zentrum für Luftund Raumfahrt, Oberpfaffenhofen, Germany, June 2008.

[31] Weiß, S., Kempe, F., and Brieß, K., "GPS Tracking on the Three-AxisStabilized Picosatellite BEESAT-4," No. N.A., $7^{\text {th }}$ Pico and Nano Satellite Workshop on Technology for Small Satellite Research, Würzburg, Germany, 2013.

[32] Ardaens, J.-S. and Gaias, G., "Angles-Only Relative Orbit Determination during the AVANTI Experiment," Proceedings of the $26^{\text {th }}$ Interna- 
tional Symposium on Space Flight Dynamics ISSFD, Japan Society for Aeronautical and Space Sciences, Japan, 2017.

[33] Gaias, G., Ardaens, J.-S., and D'Amico, S., "Formation Flying Testbed at DLR's German Space Operations Center," Proceedings of the $8^{\text {rd }}$ International ESA Conference on Guidance, Navigation $\&$ Control Systems, European Space Agency, ESTEC, Noordwijk, The Netherland, 2011.

[34] Ardaens, J.-S. and Gaias, G., "Integrated Solution for Rapid Development of Complex GNC Software," Proceedings of the Workshop on Simulation for European Space Programmes (SESP), European Space Agency, ESTEC, Noordwijk, The Netherland, 2015.

[35] Sullivan, J. and D'Amico, S., "Nonlinear Kalman Filtering for Improved Angles-Only Navigation Using Relative Orbital Elements," Journal of Guidance, Control, and Dynamics, Vol. 40, No. 9, 2017, pp. 2183-2200, doi: 10.2514/1.G002719.

[36] Newman, B., Sinclair, A. J., and Lovell, T. A., "Angles-Only Initial Orbit Determination: Comparison of Relative Dynamics and Inertial Dynamics Approaches," No. N.A., $9^{\text {th }}$ International Workshop on Satellite Constellations and Formation Flying, University of Colorado Boulder, USA, 2017.

[37] Ardaens, J.-S. and Gaias, G., "Flight Demonstration of Spaceborne Real-Time Angles-Only Navigation to a Noncooperative Target in LowEarth Orbit," No. N.A., $9^{\text {th }}$ International Workshop on Satellite Constellations and Formation Flying, University of Colorado Boulder, Colorado, USA, 2017.

[38] Gaias, G., D'Amico, S., and Ardaens, J.-S., "Angles-Only Navigation to a Noncooperative Satellite Using Relative Orbital Elements," Journal of Guidance, Control, and Dynamics, Vol. 37, No. 2, 2014, pp. 439-451, doi: $10.2514 / 1.61494$.

[39] D'Amico, S. and Montenbruck, O., "Proximity Operations of Formation Flying Spacecraft using an Eccentricity/Inclination Vector Separation," Journal of Guidance, Control and Dynamics, Vol. 29, No. 3, 2006, pp. 554-563, doi: 10.2514/1.15114.

[40] Gaias, G. and D'Amico, S., "Impulsive Maneuvers for Formation Reconfiguration using Relative Orbital Elements," Journal of Guidance, Control, and Dynamics, Vol. 38, No. 6, 2015, pp. 1036-1049, doi: 10.2514/1.G000191. 
[41] Koenig, A. W., Guffanti, T., and D'Amico, S., "New State Transition Matrices for Spacecraft Relative Motion in Perturbed Orbits," Journal of Guidance, Control, and Dynamics, Vol. 40, No. 7, 2017, pp. 17491768, doi: 10.2514/1.G002409.

[42] Woffinden, D. C. and Geller, D. K., "Observability Criteria for AnglesOnly Navigation," IEEE Transactions on Aerospace and Electronic Systems, Vol. 45, No. 3, 2009, pp. 1194-1208.

[43] Julier, S. J. and Uhlmann, J. K., "Unscented filtering and nonlinear estimation," Proceedings of the IEEE, Vol. 92, No. 3, Mar 2004, pp. 401422 .

[44] Gaias, G. and Ardaens, J.-S., "In-Orbit Experience and Lessons Learned from the AVANTI Experiment," No. N.A., 9 ${ }^{\text {th }}$ International Workshop on Satellite Constellations and Formation Flying, University of Colorado Boulder, USA, 2017.

[45] Gaias, G., Ardaens, J.-S., and Schultz, C., "The AVANTI experiment: flight results," Proceedings of the $10^{\text {th }}$ International ESA Conference on Guidance, Navigation $\&$ Control Systems, European Space Agency, ESTEC, Noordwijk, The Netherlands, 2017. 